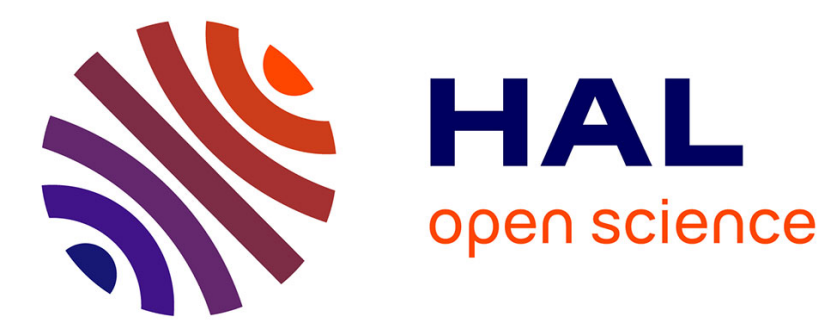

\title{
Survey on hardware implementation of random number generators on FPGA: Theory and experimental analyses
}

Mohammed Bakiri, Christophe Guyeux, Jean Couchot, Abdelkrim Oudjida

\section{To cite this version:}

Mohammed Bakiri, Christophe Guyeux, Jean Couchot, Abdelkrim Oudjida. Survey on hardware implementation of random number generators on FPGA: Theory and experimental analyses. Computer Science Review, 2018, 27, pp.135 - 153. hal-02182827

\section{HAL Id: hal-02182827 \\ https://hal.science/hal-02182827}

Submitted on 13 Jul 2019

HAL is a multi-disciplinary open access archive for the deposit and dissemination of scientific research documents, whether they are published or not. The documents may come from teaching and research institutions in France or abroad, or from public or private research centers.
L'archive ouverte pluridisciplinaire HAL, est destinée au dépôt et à la diffusion de documents scientifiques de niveau recherche, publiés ou non, émanant des établissements d'enseignement et de recherche français ou étrangers, des laboratoires publics ou privés. 


\title{
Survey on Hardware Implementation of Random Number Generators on FPGA: Theory and Experimental Analyses
}

\author{
Mohammed Bakiri ${ }^{\mathrm{a}, \mathrm{b}, *}$, Christophe Guyeux ${ }^{\mathrm{a}}$, Jean-François Couchot $^{\mathrm{a}}$, \\ Abdelkrim Kamel Oudjida ${ }^{b}$ \\ ${ }^{a}$ Femto-ST Institute, DISC Department, UMR 6174 CNRS, University of Bourgogne \\ Franche-Comté, Belfort, 90010, France \\ ${ }^{b}$ Centre de Développement des Technologies Avancées, ASM/DMN Department, Cit 20 aot \\ 1956 Baba Hassen, B. P 17,16303, Alger, Algeria
}

\begin{abstract}
Random number generation refers to many applications such as simulation, numerical analysis, cryptography etc. Field Programmable Gate Array (FPGA) are reconfigurable hardware systems, which allow rapid prototyping. This research work is the first comprehensive survey on how random number generators are implemented on Field Programmable Gate Arrays (FPGAs). A rich and upto-date list of generators specifically mapped to FPGA are presented with deep technical details on their definitions and implementations. A classification of these generators is presented, which encompasses linear and nonlinear (chaotic) pseudo and truly random number generators. A statistical comparison through standard batteries of tests, as well as implementation comparison based on speed and area performances, are finally presented.
\end{abstract}

Keywords: Random Number Generators, Field-Programmable Gate Array, Chaos, Physical security, Hardware Security, Applied Cryptography

\footnotetext{
* Corresponding author

Email addresses: mbakiri@femto-st.fr (Mohammed Bakiri), cguyeux@femto-st.fr (Christophe Guyeux), couchot@femto-st.fr (Jean-François Couchot), a_oudjida@cdta.dz (Abdelkrim Kamel Oudjida)
} 


\section{Background and Motivation}

Randomness is a common word used in many applications [1] such as simulations 2], numerical analysis [3], computer programming, cryptography 4], decision making, sampling, etc. The general idea lying behind this generic word 5 most of the times refers to sequences, distribution, or uniform outputs generated by a specific source of entropy. In other words, the probabilities to generate the same output are equal ( $50 \%$ to have "0" or "1"). If we take the security aspect, many cryptosystem algorithms rely on the generation of random numbers. These random numbers can serve for instance to produce large prime numbers which are at the origin of cipher key construction [5] (for example, in RSA algorithm [6], in Memory Encryption [7] or Rabin signatures [8]). Furthermore, when the generators satisfy some very stringent properties of security, the generated numbers can act as stream cyphers in symmetric crytosystems like the one-time pad, proven cryptographically secure under some assumptions [9]. Randomization techniques are especially critical since these keys are usually updated for each exchanged message. Even if an adversary has partial knowledge about the random generator, the behavior of this latter should remain unpredictable to preserve the overall security.

From a historical point of view, numerical tables and physical devices have provided the first sources of randomness designed for scientific applications. On the one hand, random numbers were extracted from numerical tables like census reports [10, mathematical tables [11] (like logarithm or trigonometric tables, of integrals and of transcendental functions, etc.), telephone directories, and so on. On the other hand, random numbers were extracted also from some kind of mechanical or physical computation like the first machine of Kendall and Babington-Smith [12, Ferranti Mark 1 computer system [13] that uses the resistance noise as a physical entropy to implement the random number instruction in the accumulator, the RAND Corporation [14 machine based on an electronic roulette wheel, or ERNIE (Electronic Random Number Indicator Equipment [15]), which was a famous random number machine based on the 
noise of neon tubes and used in Monte Carlo simulations [16, 17].

These techniques cannot satisfy today's needs of randomness due to their mechanical structure, size limitation when tables are used [11, and memory space. Furthermore, it may be of importance to afford to reproduce exactly the same "random sequence" given an initial condition (called a "seed"), for instance in numerical simulations that must be reproducible - but physical generation of randomness presented above does not allow such a reproducibility. With the evolution of technologies leading to computer machines, researchers start searching for low cost, efficient, and possibly reproducible Random Number 40 Generators (RNGs). This search historically began with John von Neumann, who presented a generation way based on some computer arithmetic operations. Neumann generated numbers by extracting the middle digits from the square of the previously generated number and by repeating this operation again and again. This method called mid-square is periodic and terminates in a very short

45 cycle. Therefore, periodicity and deterministic outputs that use an operator or arithmetic functions are the main difference with the earlier generators. They are known in literature as "pseudorandom" or "quasirandom" number generators (PRNGs), while circuits that use a physical source to produce randomness are called "true" random number generators (TRNGs).

50 In most cases a random number generator algorithm can be defined by a tuple $\left(S, f, g, U, x^{0}\right)$, in which $S$ is the state space of the generator, $U$ is the random output space, $f: S \rightarrow S$ is the transition mapping function, $g: S \rightarrow U$ is the output extractor function from a given state, and $x^{0}$ is the seed [18, see Figure 1. The random output sequence is $y^{1}, y^{2}, \ldots$, where each $y^{t} \in U$

${ }_{55}$ is generated by the two main steps described thereafter. The first step applies the transition function according to the recurrence $x^{t+1}=f\left(x^{t}\right)$, where $f$ is an algorithm in the PRNG case and a physical phenomenon in the TRNG one. Then, the second step consists in applying the function generator to the new internal state leeading to the output $x^{t}$, that is, $y^{t}=g\left(x^{t}\right)$. The period of 60 a PRNG is the minimum number of iterations needed to obtain twice a given output (a PRNG being deterministic, it always finishes to enter into a cycle). 


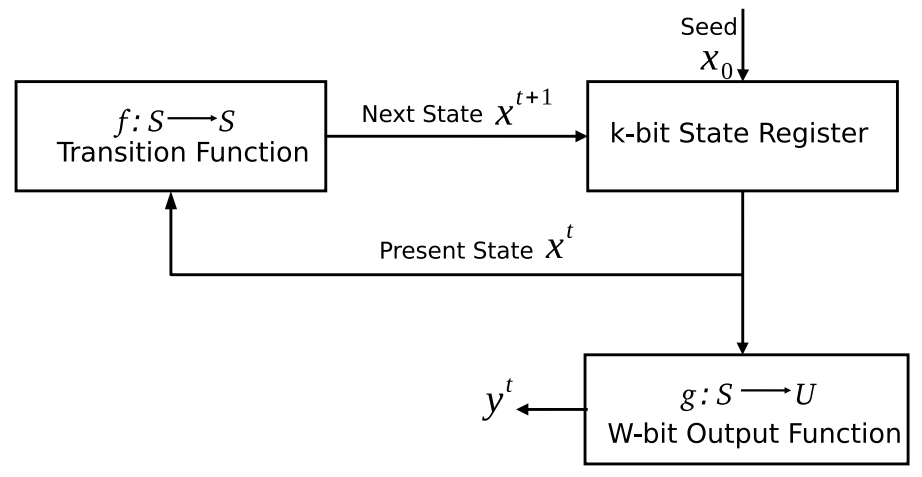

Figure 1: General architecture of a random number generator

As stated previously, the old hardware manner to build such RNGs was to use a mechanical machine or a physical phenomenon as entropy source, which can thus be based on noise [19, metastability (frequency instability [20]), semiconductor commercial or industrial component circuit (PLL 21], amplifier, inverter,...), or a variation in the CMOS/MEMS process technologies (transistor). In spite of the quality of the generated randomness, most of these techniques are however, either slow processes (i.e, extracting noise from a component) or costly (e.g., extracting or measuring noise may require specific equipment like an oscilloscope). All these previous drawbacks are the motivation behind the development of hardware generators based on a software design. The latter consist of developing deterministic algorithms by targeting a specific hardware system, like a Field Programmable Gate Array (FPGA), before automatically deploying it on the hardware architecture by using ad hoc tools.

FPGA devices are reconfigurable hardware systems. They allow a rapid prototyping, i.e., explore a number of hardware solutions and select the best one in a shorter time. The design methodology on FPGA relies on the use of a High Description Language (i.e, Verilog, VHDL, or SystemC) and a synthesis tool. Because of this, FPGA has become popular platforms for implementing random generators or complete cryptographic schemes, due to the possibility to achieve high-speed and high-quality generation of random. The general architecture of a FPGA presented in Figure 2 is based on LCA (Logic Cell Array), 


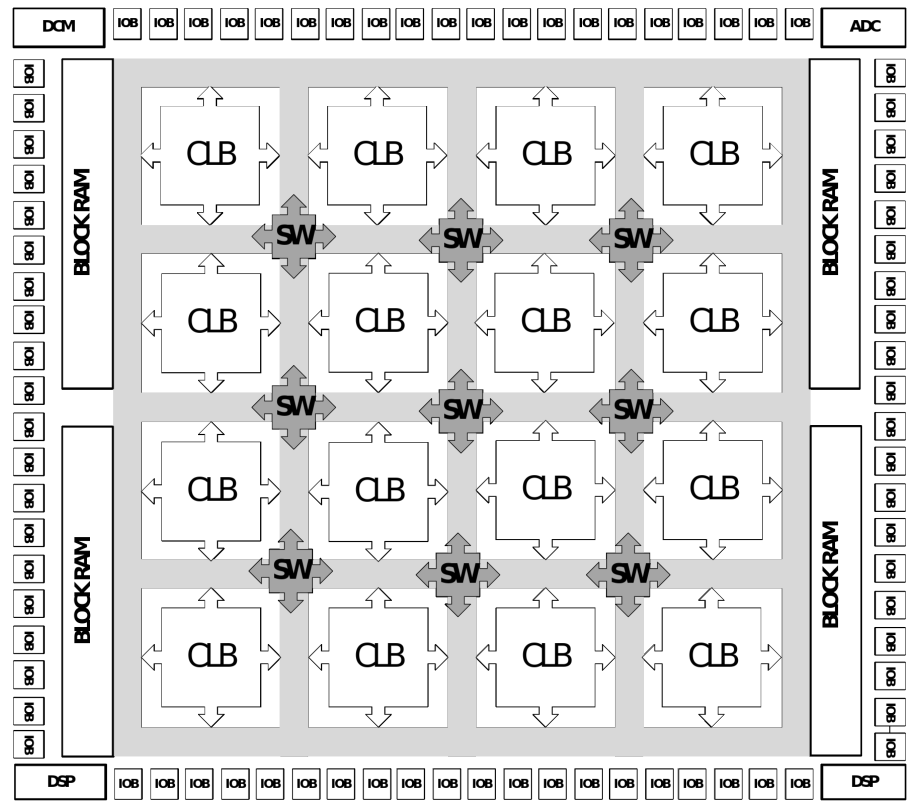

Figure 2: General structure of a Xilinx's FPGA

which is composed of three parts, namely: Configurable Logic Block (CLB) [22], Input Output Block (IOB), and interconnect switches. FPGA could addition85 ally include more complex components like a Digital Signal Processing (DSP), a Random Access Memory (RAM), a Digital Clock Manager (DCM), or an Analog-Digital Converter (ADC/DAC). The nomination of the internal blocks depends on the FPGA vendors (Xilinx, Altera, Actel ...) even they have a similar functionality. The CLB structure is mainly based on Look-Up Tables 90 (LUTs [23]), additionally with a Flip-Flop and some multiplexers. A $K$-input LUT is a $2^{K} \times 1$-bit memory array based on a truth table of $K$-bits inputs. These later can executes any logic functions as XOR/ADD/SHIFT...

Different implementations of RNG on FPGA have diverse characteristics. First of all, does it provide true random or pseudorandom numbers? In the second reproducible case, which algorithm is implemented? The next characteristic is the way each block is deployed on the FPGA, namely by computing or in a hardware manner. For instance, for a polynomial division, there is a choice 
between look-up table in software and a hardware shift register. Furthermore, the quality of the FPGA model that implements a random number generator can be evaluated according to many criteria. In a statistical perspective, the output has to be verified against some well-known test suite like the NIST [24, DieHARD [25], or TestU01 [26] ones. From the hardware perspective, one objective is to provide the highest frequency per randomly generated bit with less FPGA hardware resources (CLB, IOB, ...).

This article surveys a large set of selected hardware implementations of random number generators on FPGA. Both pseudorandom and true random generators are investigated, while linear and non-linear generators are discussed in the PRNG case. Each approach is explained in details, and a discussion on the choices of both implementations and generations are systematically given. Performance with respect to frequency, area size, weaknesses, and statistical evaluations are finally presented, when they are available.

The remainder of the article is as follows. Section 2 describes FPGA implementations of linear PRNGs, whereas the next section 3 focuses on non-linear ones. Each of these 2 sections ends by a short comparison regarding area resources and throughput frequency of the FPGA implementations. The true random ones are detailed in Section 4 while recalls regarding statistical batteries of tests and scores of some RNGs on FPGA are provided in Section 6 . This article ends by a conclusion section, in which the review is summarized and future investigative directions are outlined.

\section{Linear Pseudorandom Number Generators}

This section and the next one are devoted to pseudorandom number generators on FPGA. Recall that the latter are defined by a tuple containing a recurrent equation of the form $x^{t+1}=f\left(x^{t}\right)$. This recurrence may be linear or not. The linear case is investigated in the current section, while the non-linear case is detailed in Section 3

Linear PRNGs are a special case of linear recurrence modulo 2. They are 
convenient for low power and high speed requirement but, due to the limitation of the shift register state (two possibilities: 0 and 1), the period of these generators is usually short. Because of this, many hardware optimizations are proposed to increase the period (they will be detailed thereafter). A linear PRNG of $w$ bits can be defined by the following Equations (17) [27]:

$$
\begin{gathered}
x^{t+1}=A \times x^{t} \quad(a) \\
y^{t}=B \times x^{t} \quad(b) \\
r^{t}=\sum_{\ell=1}^{w} y_{\ell-1}^{t} 2^{-\ell}(c)
\end{gathered}
$$

Indeed the first equation $(a)$ defines the function $f$, where $x^{t}=\left(x_{0}^{t}, \ldots, x_{k-1}^{t}\right) \in$ $S=\mathbb{F}_{2}^{k}$ is the $k$-bit vector at step $t\left(\mathbb{F}_{2}\right.$ is the finite field of cardinality 2 and $S$ is the internal state space of the generator). The other equations $(b)$ and $(c)$ define the function $g$, where $y^{t}=\left(y_{0}^{t}, \ldots, y_{w-1}^{t}\right) \in U=\mathbb{F}_{2}^{w}$ is the $w$-bit output vector at step $t$, and $U$ is the state space of the output. Additionally, $A$ is a $k \times k$ transition matrix, $B$ is a $w \times k$ output transformation matrix, which produces the output bits which corresponds to the internal RNG state, and $r^{t} \in[0,1]$ is the output at step $t$. All the elements of $A$ and of $B$ are in $\mathbb{F}_{2}$.

In the simplest case we have $w=k$ and $B$ is the identity matrix, which means that the state bits are directly used as random output bits. In case where $w<k$, the output are either propagating in another circuit, or multiple state bits are XORed together to produce each output bit, as in the case of Mersenne Twister [28]. These linear generators are covering Tausworthe or Linear Feedback Shift Register [29], polynomial Linear Congruential Generators [30], Generalized Feedback Shift Register (GFSR [31]), twisted GFSR [32], Mersenne Twister, linear cellular automaton, and combinations between them. More details will be presented regarding each of these generators in this survey. 


\subsection{Linear Congruential Generators}

Linear Congruential Generators (LCGs) [30] are founded on system of linear recurrence equations defined as:

$$
x^{t+1}=\left(a x^{t}+b\right) \bmod 2^{k}
$$

where $a$ (the "multiplier"), $b$ (the "increment"), s.t. $0 \leq a, b \leq 2^{k-1}$ are parameters of the generator,

This latter is often called a Multiplicative Congruential Generator [33] (MCG) if $b=0$, and Mixed Linear Congruential Generator, otherwise.

150

In 34, two optimized LCGs are proposed, namely the Ranq1 and Ran 35. Ranq1 is a MCG working modulo $2^{64}$, while its seed is produced by a 64 -bits right $X O R$ shift [36]. Let us first recall that the $X O R$ shift takes an input and iteratively executes an exclusive or (XOR) of the binary number with a bit shifted translation (left and right) of itself. The second one, the Ran generator, combines a LCG generator with two XORshifts, and the results are XORed by a Multiply with Carry (MWC) generator [37]. In MWC, the equation (2) is modified as follows: the constant $b$ is replaced by the carry $b^{t}$ which is defined by $b^{0}$, the initial carry, is less than $a$ and $b^{t+1}=\left\lfloor\frac{a x^{t}+b^{t}}{2^{32}}\right\rfloor$.

Authors of 34 optimized the implementation of the 64 -bits constant coefficient multiplier $a \times x^{t}$. However the 64-bit multiplication is problematic due to DSP macro limitations that support only 18-bit operations in Xilinx's FPGA. This is why these authors proposed a pipeline of multiplier-adder architecture, which takes 5 cycles for Ran and 4 for Ranq1, while the output is the least significant 32 bits. Comparisons realized in their article showed that these two new optimized implementations have a lower cost in the area than other PRNGs like the Mersenne Twister [28, which use memories or multiplier macros of the FPGA. But the authors were wrong when they assumed that the multiplication by a constant is similar to the multiplication by a variable. In the former (Oudjida et al [38, 39, 40]), the multiplication is implemented in a multiplierless way, 
Authors of [41] have presented a coupling of two Coupling Linear Congruential Generators (CLCG), further denoted as CLCG-1 and CLCG-2. Each one generates a separate output with different parameters described as follow:

$$
\begin{aligned}
& x_{1}^{t+1}=\left(a_{1} x_{1}^{t}+b_{1}\right) \quad \bmod 2^{k} \quad x_{3}^{t+1}=\left(a_{3} x_{3}^{t}+b_{3}\right) \bmod 2^{k} \\
& x_{2}^{t+1}=\left(a_{2} x_{2}^{t}+b_{2}\right) \quad \bmod 2^{k} \quad x_{4}^{t+1}=\left(a_{4} x_{4}^{t}+b_{4}\right) \bmod 2^{k} \\
& C_{1}^{t+1}=\left\{\begin{array}{ll}
1 & \text { if } x_{1}^{t+1} \geqslant x_{2}^{t+1} \\
0 & \text { otherwise. }
\end{array} \quad C_{2}^{t+1}= \begin{cases}1 & \text { if } x_{3}^{t+1} \geqslant x_{4}^{t+1} \\
0 & \text { otherwise. }\end{cases} \right.
\end{aligned}
$$

The first CLCG-1 of [41] is characterized by $\left\{x_{1}^{t+1}, x_{2}^{t+1}, C_{1}^{t+1}\right\}$ while the second CLCG-2 is defined with $\left\{x_{3}^{t+1}, x_{4}^{t+1}, C_{2}^{t+1}\right\}$ ( $C_{1}^{t}$ and $C_{2}^{t}$ are bit sequences). CLCG-2 aims at selecting which bit must be taken from CLCG-1 as a final output $y^{t}: y^{t}=C_{1}^{t}$ if $C_{2}^{t}=0$, otherwise the bit $C_{1}^{t}$ is ignored. For instance, the authors assume a simple format of the multipliers: $a_{1}=a_{3}=2^{\delta_{1}}+1$ and $a_{2}=a_{4}=2^{\delta_{2}}+1$, where $1<\delta_{1}, \delta_{2}<k$. Indeed, the new format of $x_{1}^{t+1}$ for CLCG-1 (and similarly for $x_{2}, x_{3}$, and $x_{4}$ ) is as follow:

$$
x_{1}^{t+1}=\left(\left(2^{\delta_{1}} \times x_{1}^{t} \quad \bmod 2^{k}\right)+x_{1}^{t}+b_{1}\right) \bmod 2^{k},
$$

where $2^{\delta_{1}} \times x^{t}$ is the result of shifting $x^{t}$ exactly $\delta_{1}$ times to the left, and the modulation is computed as the $k$ least significant bits of what is in parentheses. However, a large value of $k$ leads to a large latency. To solve this problem, an implementation of $P$ stages of addition and comparison for the two CLCGs has been proposed in this article. It divides the $k$-bits numbers into $P$-pipeline parts, processes each $k / P$-bits part in a pipeline stage, and finally generates 1bit of $C_{1}$ and $C_{2}$ simultaneously. Additionally, it takes the results of each stage and sends them to both the previous and the next stages, in order to produce the current and the next outputs.

\subsection{Linear Feedback Shift Register generators}

Linear Feedback Shift Register generators (LFSR) or Tausworthe [29] are linear recurrent generators. An LFSR uses a sequence of shift registers to generate one bit per iteration. Each register is connected to its neighborhoods, the 


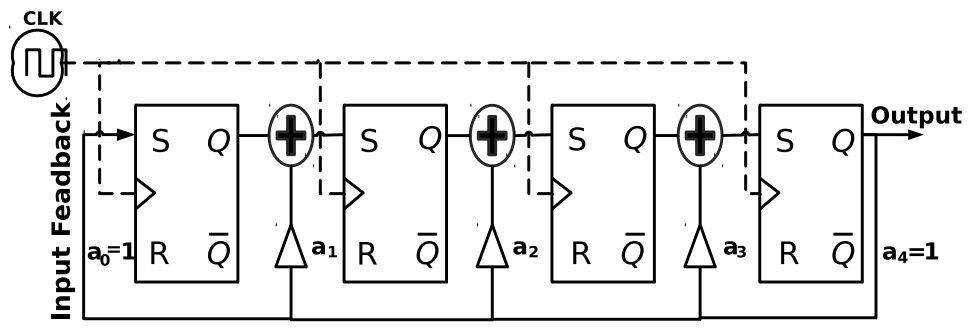

(a)A 4-bits Galois LFSR architecture

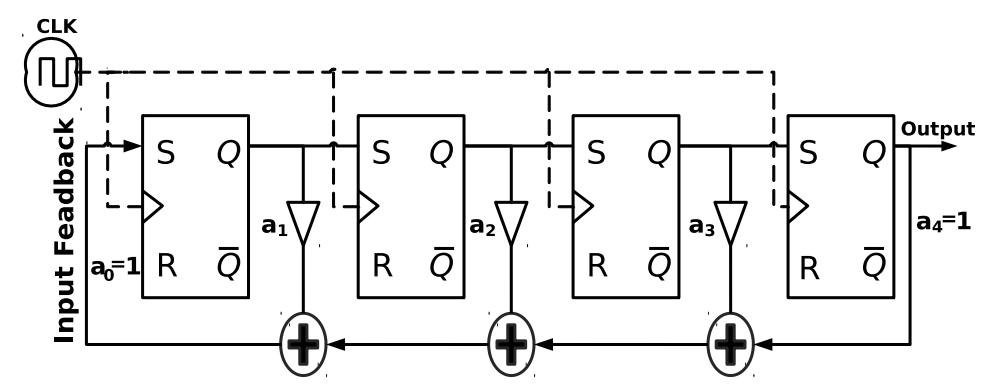

(b)A 4-bits Fibonacci LFSR architecture

Figure 3: A 4-bits linear feedback shift register generator with a feedback polynomial $a_{0} * X^{4}+a_{1} * X^{3}+a_{2} * X^{2}+a_{3} * X+a_{4}\left(a_{0}=a_{4}=1\right)$.

binary value in each register is shifted at each iteration, while the last register produces the output (see Figure 3). A XOR is operated on some designed registers to build a feeadback input to the first register, which is expressed by a characteristic polynomial. As depicted in Figure 3 two configurations are usually considered, namely the Galois and Fibonacci setups. The matrix $A$ of Eq. (1) is in this case:

$$
A=\left(\begin{array}{cc}
0 & I_{k-1} \\
a_{k} & a_{k-1}, \ldots, a_{1}
\end{array}\right) .
$$

The characteristic polynomial of the matrix $A$ is $x^{t+1}=a_{1} x^{t}+\cdots+a_{k} x^{t+1-k}$. In the above equations, $a_{1}, \ldots, a_{k}$ represent the LFSR coefficients, each in $\mathbb{F}_{2}$. Accordingly, if any of these coefficients exists, it deploys a XOR operand on the output (remark that the matrix $B$ of Equation (1) is the identity matrix $I$ ).

Even though many FPGA implementations of such LFSRs can be found 
In [42, the authors present two types of LFSRs. The first one, called Shrinking Generator (SG), it uses two LFSRs of 67 bits (LFSR-1 and LFSR-2). At each clock cycle, the SG directly takes the value of the output bit which is generated by the second LFSR-2 if the output bit from the first LFSR-1 is equal to 1 ; if not, both outputs are discarded. The second version, named Alternating Step Generator (ASG), considers a third LFSR-3 of 141 bits in addition of the two previous ones. This latter is used to control which output bit will be taken from the two first LFSRs of 131/137-bits. For comparison purposes, if $T_{1}, T_{2}$, and $T_{3}$ are the periods of LFSR-1, LFSR-2, and LFSR-3 respectively, let us note that the SG has a total period of $T_{S G}=\left(2^{T_{1}}-1\right) \times\left(2^{T_{2}}-1\right)$ (length $\simeq 64$ bits), while it is $T_{A S G}=2^{T_{1}}\left(2^{T_{2}}-1\right) \times\left(2^{T_{3}}-1\right)$ for ASG (length $\simeq 128$ bits $)$.

LFSR based Accumulator Generator proposed in [43] is a PRNG based on Digital Sigma-Delta Modulator 44 made from accumulator circuits. This latter is used to divide the frequency in a Fractional-n Frequency Synthesizer [45]. Authors of [43] propose a pipeline of $N=9$ serial digital accumulators of $w=8$ bits based FPGA as described in Figure 4. Each accumulator, which can produce $M=2^{w}$ possible values, is a self-recursive structure based on quantization error mapping function formalized in Eq. (8), where the accumulator's feedback coefficients are time-varying, using another linear feedback shift register. The accumulator, which is presented in Equation (7), is parameterized by the input seed $x^{0}$, the accumulator sum $p$, the carry output $a c c u$, the quantization error $e$, and the feedback coefficients $c=2^{-w}$ of the accumulators as outputs. The input of each $n=0, \ldots,(N-1)$ stage during the processing uses the quantization error $e^{t-1}$ of the previous stage. Therefore, the PRNG gives a better uniform outputs by propagating the latter $(e)$ at all stages following the Equation (7). The accumulator feedback coefficient $c$ is implemented with another accumulator. The latter are multiplied by a binary variable $d_{w} \in\{0,1\}$ of the LFSR to control the feedback depending on the period of LFSR. The final output 


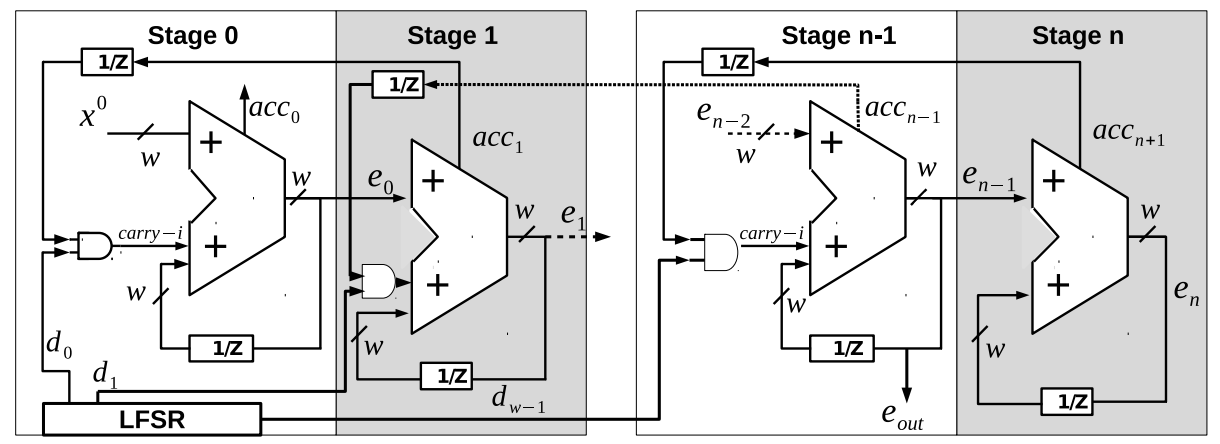

source 43$]$

Figure 4: Block-level model of a w-bit digital accumulator PRNG comprising $n$ stages

$y^{t}=e_{\text {out }}$ is the last generated $e_{N-1}^{t}$ evaluated in Equation (8).

$$
\begin{gathered}
p_{n}^{t+1}= \begin{cases}x^{0}+e_{0}^{t}+a c c u_{1}^{t} d_{0}^{t}, & n=0 \\
e_{n-1}^{t+1}+e_{n}^{t}+a c c u_{n+1}^{t} d_{w-1}^{t+1}, & 0<n<N-1 \\
e_{n-1}^{t+1}+e_{n}^{t}, & n=N-1\end{cases} \\
e_{n}^{t+1}=p_{n}^{t+1} \bmod 2^{w} \text { and } a c c u_{n}^{t}=\left\{\begin{array}{l}
1 p_{n}^{t} \geq M \\
0 p_{n}^{t}<M
\end{array}\right.
\end{gathered}
$$

\subsection{Look-up Table Optimized Generators}

Look-up Table (LUT [23]) optimized generators use logic block as a digital component defined in a CLB provided by the FPGA vendors. It is used for implementing many function and operation generators for a hardware optimization purpose. A LUT consists of a block of RAM (Random Access Memory) implemented as a truth table that is indexed by the LUT inputs.

In [46, 47, 48, the authors present a series of LUT PRNGs based on $\mathbb{F}_{2}$ linear matrix recursive algorithms (see Figure 5). The main idea is to produce a maximum efficiency at area level. The authors associate either Flip-Flops (FF), Shift Registers (SR), or block of RAMs (RAM) with LUT to perform shift/multiplication operations in FPGA. However, creating long period se- 
quences $T=2^{w}$ with this method is a difficult task. To solve this problem, words: the first two words $x^{t}$ and $x^{t+1}$ with the median word $x^{t+m}$. More precisely (see Figure 6): 1. It computes the $c$ least significant bits (LSB) of $x^{t+1}$ and the $k-c$ most

The first proposed PRNG is called LUT-OPT (LUT optimized, $(a)$ in Figure 5. It maps each row of the recurrence matrix $A$ as a $X O R$ gate using just LUT and FF. To generate $w$ bits per cycle requires $w$ LUT-FFs in a single LUT of $k$-bits during a period of $T=2^{w}$ where $w=k$ (see Figure 5). Their estimations of the FPGA resources conclude that even if an application requires 64 bits for each cycle, their implementations necessarily use 512 LUT-FFs to produce a period of $2^{512}-1$. The second one, the LUT-FIFO $(b)$, is used to increase the period up to $T=2^{(w+k * L)}$ without using the pair LUT-FF, which uses RAM block memory (dual-port RAM) of FPGA as $L \times k$ FIFO to store the recursive sequences. In this case, each new output bit is depending on one bit from the last iteration. They next propose a FIFO based shift-register SR (c) with a fixed length $L$ of 1-bit, to load the $w$-bit state in parallel instead of using dual-port RAM. They also propose a LUT-SR PRNG $(d)$ that turns the use of LUT as a $k$-bit Shift-Register using "Xilinx SRL32", with the length of each "SR" varying as follows: $1<L_{i}<L$. The "Xilinx SRL32", allows the cascading of any number up to 32-bit shift registers to create a shift register with any size needed.

\subsection{Twisted Generalized Feedback Shift Register PRNG}

Twisted Generalized Feedback Shift Register (TGFSR) proposed in 32] is an extension of Generalized Feedback Shift Register "GFSR" [31, which uses an array of shift registers to generate more than one bit for each state change. Therefore, a TGFSR is based on recurrence of $N$ sequences of words, $x^{0}, \ldots$, $x^{N-1}$, each containing $k$-bits and two parameters, namely a bitmask size $c$, such that $c \leqslant k-1$ and a initial median position $m$ with $1 \leq m \leq N$.

TGFSR computes the $t+N$-th word $(t=0,1, \ldots)$ by operating with three 


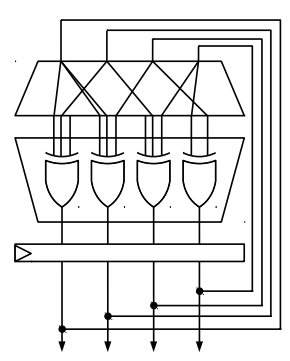

(a) LUT-OPT $T=2^{w}$
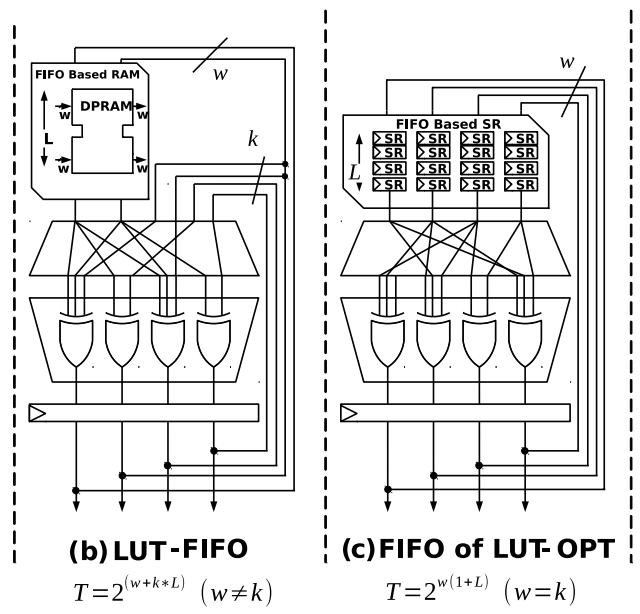

$T=2^{w(1+L)}(w=k)$

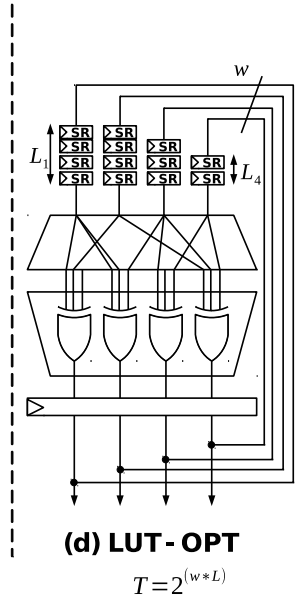

$T=2^{(w * L)}$

source 48

Figure 5: LUT based shift-register and FIFO FPGA optimized PRNG: (a) maps each row of the recurrence matrix as a XOR gate using LUT-FF, (b) uses RAM block memory as $k \times k$ FIFO to store the recursive sequences, (c) loads the state in FIFO based shift-register SR instead of BRAM, (d) cascading of any number of Xilinx SRL32 to create a $k$-bit SR

significant (MSB) ones of $x^{t}$. These two vectors are obtained thanks to the two following bitmask vectors: $\overline{S_{c}}$ for $(0, \ldots, 0,1, \ldots, 1)$ and $\underline{S_{k-c}}$ for $(1, \ldots, 1,0, \ldots, 0)$.

2. These two vectors are further concatenated through $\left(x^{t} \& \underline{S_{k-c}}\right) \mid\left(x^{t+1} \&\right.$ $\left.\overline{S_{c}}\right)$.

3. The result $x^{\prime}$ is then "multiplied" with a matrix $A$, caracterized with values $\left(a_{0}, a_{1}, \ldots, a_{w-1}\right)$ as defined in Eq. 10 .

4. The final results of the previous calculations are then XORed with the median word $x^{t+m}$.

By putting $c=0$, then the Equation (9) represents the TGFSR PRNG 32], conversely it is Mersenne Twister [28.

$$
x^{t+N}=x^{t+m} \oplus\left(\left(\left(x^{t} \& \underline{S_{k-c}}\right) \mid\left(x^{t+1} \& \overline{S_{c}}\right)\right) \times A\right) \text {, where }
$$




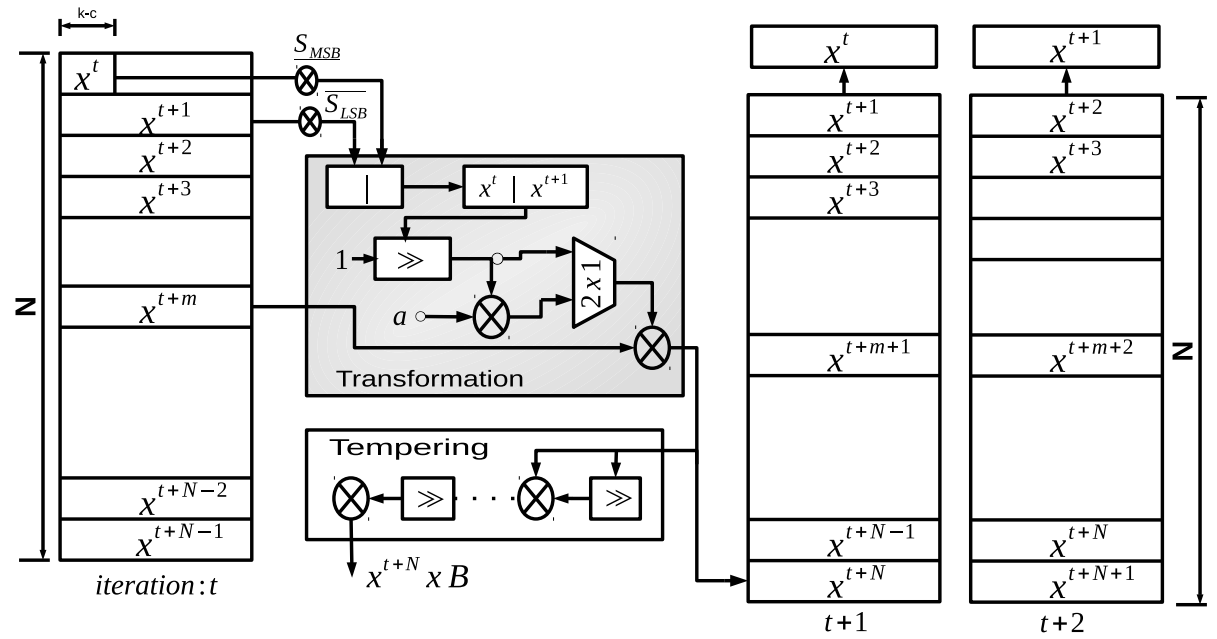

Figure 6: Twisted Generalized Feedback Shift Register architecture: at each recurrence operation $t$, it computes $x^{t+N}$ thanks to the three words $x^{t}, x^{t+1}$, and $x^{t+m}$ and generates the output with tempering function

$$
x^{\prime} \times A= \begin{cases}x^{\prime} \gg 1 & \text { if } x_{0}^{\prime}=0 \\ \left(x^{\prime} \gg 1\right) \oplus\left(a_{0}, a_{1}, \ldots, a_{w-1}\right) & \text { otherwise }\end{cases}
$$

Consider $c_{1}$ and $c_{2}$ as given bitmasks and $b_{1}, b_{2}, b_{3}$, and $b_{4}$ are constant integer parameters. At iteration $t$, TGFSR uses a tempering module to improve the equidistribution. This step, which is described a sequence of bitwise/shift computation is equivalent to a matrix product as formaized in Eq. (1)(b).

This one is defined in Equation (11) where $c_{1}, c_{2}$ (resp. $b_{1}, b_{2}$ ) are tempering bitmasks (resp. bit shifts).

$$
\begin{aligned}
& z=x^{t+N} \oplus\left(x^{t+N} \gg b_{1}\right), \\
& z=z \oplus\left(\left(z \ll b_{2}\right) \& c_{1}\right), \\
& z=z \oplus\left(\left(z \ll b_{3}\right) \& c_{2}\right), \\
& y^{t}=z \oplus\left(z \gg b_{4}\right) .
\end{aligned}
$$

Mersenne Twister (MT) is proposed as a special case of TGFSR that has a long period of $2^{w N-c}-1$. To achieve this, the authors in [28] propose two MT 
configurations:

- "MT11213" with a period of $2^{11213}-1$ that has $w=32, N=351, m=$ $175, c=19$, and $a=0 x E 4 B D 75 F 5$ as recurrence parameters, and $c_{1}=$ $0 x 655 E 5280, c_{2}=0 x F F D 58000, b_{1}=11, b_{2}=7, b_{3}=15$, and $b_{4}=17$ for tempering ones

- "MT19937", which has a period of $2^{19937}-1$, has $w=32, N=624$, $m=397, c=31$, and $a=0 x 9908 B 0 D F$ as recurrence parameters, and $c_{1}=0 x 9 D 2 C 5680, c_{2}=0 x E F C 60000, b_{1}=11, b_{2}=7, b_{3}=15$, and $\left.b_{4}=18\right]$ for Tempering ones.

Two FPGA implementations of Mersenne Twisters MT19937 and MT11213 are proposed in 34 for Monte Carlo applications in finance. The authors implement many Block RAM memory or namely "BRAM" for matrix multiplications: a single dual-port BRAM for MT11213 and two dual-port BRAM for MT19937. The RAM memory, configured in the read-before-write mode, operates like a feedback shift register. In this mode, the new inputs are stored in memory at appropriate write address, while the previous data are transferred to the output ports. This latter coming from BRAM are then processed following the Equation (6). The same approach has been proposed in [4] for MT19937 using 2 BRAM. Authors of [50], for their part, have implemented the MT11213 in three platforms for the sake of comparison, namely: FPGA, CPU, and GPU. Remark that, for testing the FPGA performances, initial and Tempering matrix parameters have been extracted from a PC software, due to the hardware cost consuming by the initialization stage of MT. However, both transformation and Tempering modules are executed in FPGA. In this case, two dual-port BRAMs are necessary for the other stages. This structure reduces the area compared to other MT implementations in FPGA, and the speed up is about $\approx 9 \times$ and $\approx 25 \times$ compared to GPU and $\mathrm{CPU}$ respectively.

In [51], the authors have proposed two parallel PRNG implementations with many levels of three different Mersenne Twisters: the MT19937-32bits, the MT19937-64bits, and the SIMD-oriented Fast Mersenne Twister SFMT19937 [52]. 
The first one is the Interleaved Parallelization (IP), that generates $w$-bits for each $P$ memory block separately.

In the IP configuration, the $N=624$-words state vector is located across multiple memory banks of the same size. Each $P$ memory bank has $d$ input/output ports I/O of $w$-bits, while each I/O port generates $v$-bits per clock cycle every $q$ read operation. Therefore, the number of clock cycle $\tau$ required to generate a random number is equal to $\tau=(w *(q+1)) / v * d$. The second one is the Chunked Parallelization (CP), that uses the output bits of each RAM bank as the far recurrence input for the next RAM bank. Therefore, the $N$-words state vector is sequentially split into chunks across a number of banks of different size. Even though the IP version has a better throughput than the CP one, the latter uses lesser RAM blocks compared to the IP version (3 levels of CP use 2 BRAMs while IP uses 3).

Authors of [53] give more hardware details for the deployment of RAM memories. Their MT19937 implementation consists of a transform unit, a Temper Unit, a control unit "Crossbar" implemented using 7 multiplexers, a 3R/1W RAM, and an address unit for RAM access ( 3 read addresses and 1 address for writing). The main key of the latter is the implementation of 624 states of 32-bits register using BRAM memory of the FPGA (see Figure 7). Therefore, instead of fetching the 3 state vectors using 3 BRAM as in [51], two dual-port BRAMs of $312 \times 32$-bits can perform in each cycle 3 read operations and 1 write one. The $R / W$ for the first BRAM operates with an even address, while the second $R / W$ is in the odd address.

In [54, various degrees of parallelization of the MT19937 architecture (of degrees 2, 3, 4, and 6) implemented in [53] and used for Monte-Carlo based simulations are proposed. In this case, the configuration of BRAM is the key of the parallelism, where each degree corresponds to the number of BRAM that are used ( 4 degrees $=4$ implemented RAMs). The authors use one $206 \times 32$-bit, two $207 \times 32$-bit dual-port BRAM, and four registers to provide state consistency 320 for the given parallelized states for 3 degrees as an example. Here, all I/O ports of three BRAM are in read mode during initialization, while in the runtime just 


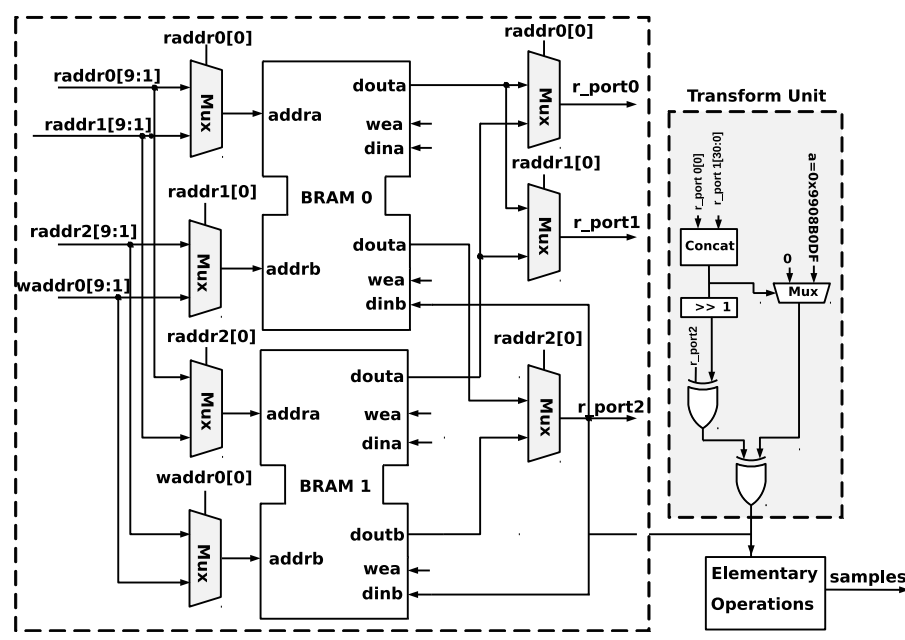

source 53 ]

Figure 7: Mersenne Twister MT19937 architecture using 3R/1W BRAM: at each cycle, $R / W$ address is even address for BRAM0 and odd for BRAM1

one is in read mode (the others being in write mode).

Finally, a recent FPGA implementation of Mersenne Twisters is presented in [55]. The authors propose an alternative solution of the use of RAMs, which is named Circular Buffer (CB). It is implemented for MT19937 (see Figure 8). The solution is based on the fixed relationship between word indices. Words $x_{j}^{t}$, $x_{j+1}^{t}$, and $x_{j+m}^{t}$ written in the buffer are passed to the transform unit. At each iteration, the first word $x_{j}^{t}$ is clocked out of the buffer while new data $x_{j}^{t+1}$ is written to the free location. By this way, the linear recurrence and the buffer of registers can be considered as a circular buffer. The linear recurrence is carried out by some combinational logic between the input and the output of the buffer. Therefore, the architecture is simplified since no logic operation for the table indices is needed.

\subsection{Cellular Automata based PRNGs}

Cellular Automata (CA) is a discrete model, proposed by John von Neumann and Stan Ulam [56] as formal models of self-reproducing robots. The basic representation of one dimensional CA PRNG includes $N$ cells with an internal 


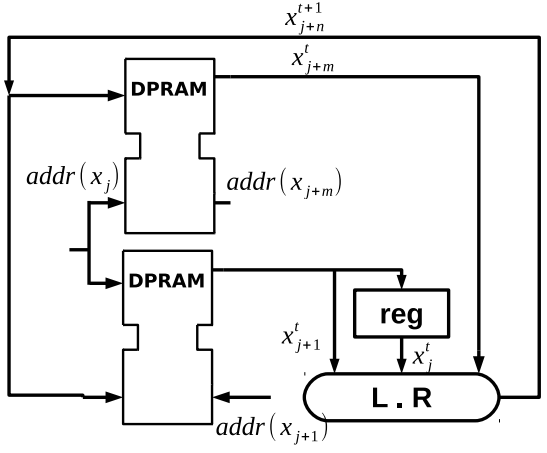

(a)BRAM configured as $3 \mathrm{R} / 1 \mathrm{~W}$

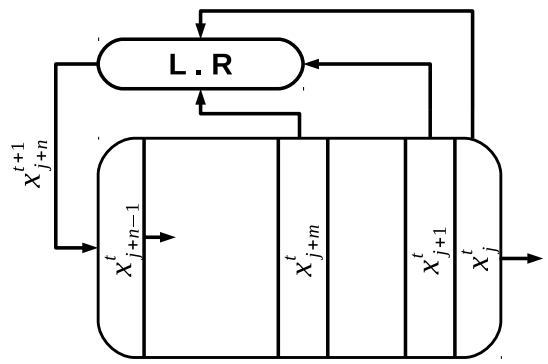

(b)Circular Buffer

source [55]

Figure 8: Different deployments of the linear recurrence (L.R) for Mersenne Twister PRNG: (a) using BRAM configured as 3R/1W, (b) using Circular Buffer of registers (L.R is linear recurrence of transferring function of $\mathrm{MT}$ )

state machine that can be a Boolean function rule and $k=1$-bit output as described in Equation 12 . The latter consider the function $f:\{0,1\}^{N} \rightarrow\{0,1\}$ as the local transition rule, and the cells neighborhood size $N$ is $2 * r d+1$, where $r d$ is the radius that represents the standard 1-D cellular neighborhood. Therefore, at each iteration $t$, the CA structure can hold and update the internal state for each cell, depending on the local rules and the states $x^{t} \in\{0,1\}$ of their neighborhoods $j(j=1, \ldots, N)$. There are $2^{N}(r d=1$ and $N=3)$ states for a single CA producing $256\left(2^{8}\right)$ possible rules classed by the Wolfram code [57.

$$
x_{j}^{t+1}=f\left(x_{j-r d}^{t} \ldots x_{j}^{t} \ldots x_{j+r d}^{t}\right)
$$
The 184 rule updates the middle bit $x_{j}^{t}$ and then left shifts the input in the next iteration $t$ as follows: $f(111)=1, f(110)=0, f(101)=1, f(100)=1$, $f(011)=0, f(010)=0, f(001)=0, f(000)=0$ (i.e. $11101011 \rightarrow 101011)$.

Hybrid CA generator (HCA) is defined with more than one rule and can 340 Each transition cycle has a $2 \times 2^{N}$ length cycle. Two hybrid CAs are proposed in [58] as part of an encryption system. The first one is a PRNG of single state 
transition using rules 90 , defined by $f\left(x_{1}^{t}, x_{2}^{t}, x_{3}^{t}\right)=x_{1}^{t} \oplus x_{3}^{t}$, and 150 , defined by $f\left(x_{1}^{t}, x_{2}^{t}, x_{3}^{t}\right)=x_{1}^{t} \oplus x_{2}^{t} \oplus x_{3}^{t}$ to generate an encrypting real-time key stream. The second one is a block cipher of two state transitions, each having 8 cycles length with 51/153/195 rules. The aim of this application is to use the first HCA to select the transition sequences between rules used by each cell of the second HCA in the block cipher. The FPGA implementation of each CA is done with a logic combinational circuit (LCC) to define the rules. Then it uses LCG to control the loading operation of the CA and stores the data into a D flip-flop. Authors of [59], for their part, create an automatic software tool to generate the RTL code of any HCA configuration. Finally, in [60, authors increase the ratio of frequency/area and the security of their previous PRNG [59] by using a chain of HCAs instead of a single one.

Mixed CA generator is proposed in [61, where the author mixes the outputs of a 37-bits hybrid CA (rules 90 and 150) with a 43-bits LFSR to obtain a large period. However, some drawbacks of this implementation are revealed during statistical evaluation, which can be surpassed only if the two PRNGs are clocked at different clock frequencies. This is why a new solution is presented in 62 . In this article, authors propose to XOR the last bit of HCA with the last bit of LFSR to generate 1-bit per clock cycle. As a repercussion, they found that the optimal combination for a PRNG of high quality is 16-bits for CA with a 37-bits LFSR.

Self-Programmable CA (SPA) was presented first as a new rules for CA generator in 63]. The topological behavior of the generator proposed in [64] is the use of a super-rule 90/156 to dynamically determine when the rules have to change in each CA cell (see Figure 9). In practice, the input rules of each neighbor cell are also a second $\mathrm{CA}$ which is executed in parallel with the main cellular automata. Remark that, despite SPA gives a better throughput than 370 the LFSR/HCA combination PRNG 62, it fails to pass the statistical tests of DIEhard battery.

Another cellular automata based PRNG is proposed in 65. This latter combines a CA with a Non-LFSR (NSFR) generator based on A2U2 stream 


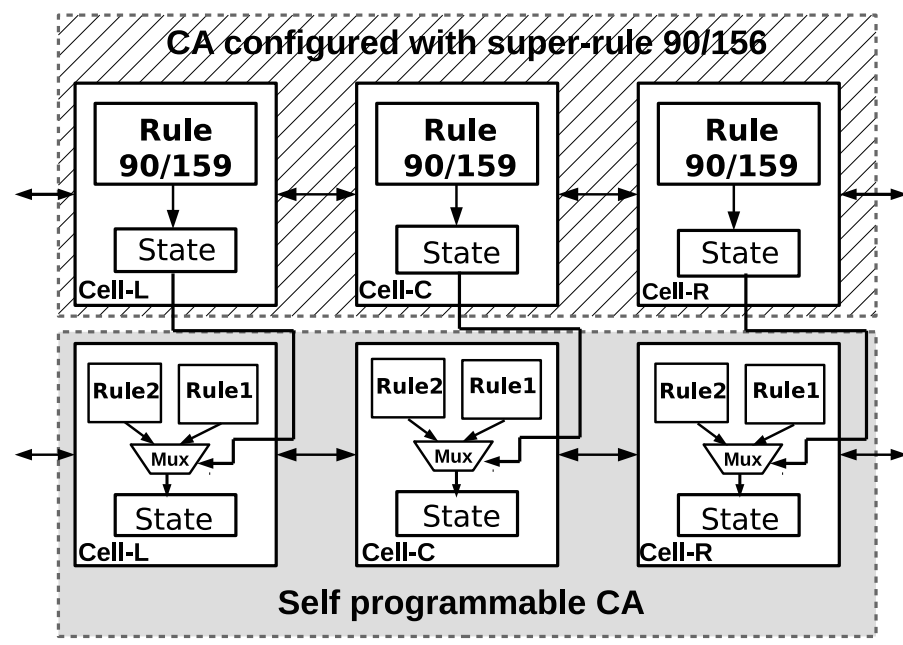

source 63

Figure 9: Self-Programmable cellular automata generator: uses a super-rule 90/156 to dynamically determines when the rules have to change in each CA cell

cipher design. Recall that the stream cipher A2U2 [66] was presented as a new key cryptographic generator of 56-bits for RFID tags application. It has a LFSR counter of 7-bits and two non-linear feedback shift registers (NFSRs, 17 and 9-bits). However, NFSR is known for its short period length. Hence, their main contribution is to associate a CA PRNG of 9-bits to increase the period of NFSR, both having feedback between them (which means that the seed of NFSR is provided by CA and vise versa). This approach improves resistance to various forms of cryptanalysis like correlation attacks and algebraic ones. For the sake of completeness, notice that the authors of [67 have proposed a different implementation concept of the usual rules in CA. In their proposal, the initial state configuration of $\mathrm{CA}$ and its length depend on the current date.

\section{Non-Linear Pseudorandom Number Generators}

\subsection{Blum Blum Shub based PRNGs on FPGA}

Blum Blum Shub generator (BBS) proposed in 68 is a non-linear and cryptographically secure PRNG based on the quadratic residue problem $x^{2}=q$ 
$\bmod w$, where $q$ is the "quadratic residue". It works as follows: consider $n=p \times q$, where $p$ and $q$ are prime numbers that are congruent to $3 \bmod 4$. Let $x^{0}$ be an integer lower than $w$, which operates as a seed of the BBS generators. Consider now the recurrent sequence $x^{t+1}=\left(x^{t}\right)^{2} \bmod w$, and $j=\left\lfloor\log _{2}\left(\log _{2}(w)\right)\right\rfloor$, where $\lfloor x\rfloor$ is the integral part of $x$. Then, at iteration $t$, the BBS generator outputs the $j$ least significant bits of $x^{t}$.

Despite its cryptographic security, only a few FPGA implementations of BBS can be found in the literature. They are listed hereafter. In 69, the authors present an area comparison without any optimization between a 4-bits LFSR and a 16-bits BBS PRNG. Another proposal is provided in 70 for RFID tag applications. In this article, the authors present a FPGA implementation of BBS with $n$ ranging from 160 to 512-bits. They consider various modular multiplication algorithms to optimize the main BBS equation. The Montgomery [71 iterative approach exhibits the lowest cost area. In [72, the authors propose a hybrid RNG of an off-chip TRNG based on Ring Oscillator 4 and a BBS PRNG generator implemented in FPGA. The TRNG generates the first random clocked by a RC circuit used as a seed for BBS. Then, the BBS uses an ALU structure to implement the squaring and modulo operations.

\subsection{Chaotic PRNG}

Chaotic generators (CPRNGs) are non-linear generators of the form $x^{0} \in \mathbb{R}$ : $x^{t+1}=f\left(x^{t}\right)$, where $f$ is a chaotic map. They are attractive applications of the mathematical theory of chaos. Reasons explaining such an interest encompass their sensitivity to initial conditions, their unpredictability, and their ability of reciprocal synchronization [73. From a cryptographer point of view, these chaotic PRNGs have major drawbacks often reported [74].

Chaotic Mapping PRNG are based on a polynomial mapping that uses a nonlinear dynamic transformation, which is a quadratic mapping. Most of these generators are based on the Logistic Chaotic Map called also "LCG" map [75], defined as follows: $x^{t+1}=\alpha \times x^{t}\left(1-x^{t}\right)$, where $0<x^{t+1}<1$ and $\alpha$ is the biotic potential $(3.57<\alpha<4.0)$. The logistic map is mainly depending on the 
parameter $\alpha$ : its chaotic behavior is lost when $\alpha$ is out of the range provided above. Moreover, if $\alpha>4$ and for almost all initial values, the outputs diverge and leave the interval $[0,1]$. The second most frequently used function is the Hénon chaotic map [76], which takes a point $\left(x^{t}, y^{t}\right)$ within the plan square unit and maps it into a new point $\left(x^{t+1}, y^{t+1}\right)$. This map is defined by these equations: $x^{t+1}=1+y^{t}-a\left(x^{t}\right)^{2}$ and $y^{t+1}=b x^{t}$, where $a$ and $b$ are called canonical parameters.

In [77], the authors have used fixed point representation [78] to implement the logistic map using Matlab DSP System Toolbox software. Fixed-point format is an approximation of real numbers, with much less precision and dynamique range than the floating-point format. Nevertheless, it has the merit of being very efficient in high-speed and low-power applications. This unit requires less power and cost to manipulate such kind of numbers than usual floating-point circuitry. They generate many designs with different lengths from 16 to 64 bits, where the resources are depending on the precision (24 to 53 bits). The multiplication is implemented with DSP blocks of FPGA that perform $18 \times 25$ bits multiplications, while the multiplication by a constant $\alpha$ is a simple series of add and left-shift operations.

Authors of [79] compare the implementation of logistic map with the Hénon one. Unlike the logistic map, the 64 bits multiplication in Hénon [76] map cannot be implemented with a left shift operation, which leads to the use of DSPs blocks 440 of the FPGA for all multiplications needed to implement $a x^{2}$. Two optimized versions of PRNGs based on chaotic logistic map are proposed in [80, which aim to reduce resources and increase frequency, unlike in [77, 79]. The first one is based on LUT and DSP blocks of the FPGA. The second one rewrites the logistic map equation as follows: $x^{t+1}=\alpha x^{t}-\alpha\left(x^{t}\right)^{2}$. The objective of these two PRNGs is to pipeline the multiplication operations and synchronize them while adding some delays into each stage, in order to ensure a parallel execution of sequences. The outputs are generated for each 8-16 clock cycles and in each cycle a new seed is inserted.

In [81, the authors vary the biotic potential $\alpha$ and observe the divergence 

extension of chaotic maps. It is also spatially chaotic (many mathematical models can be used to represent this type of generator). For instance, in [86] the authors present a spatiotemporal chaotic PRNG, which is based on a coupled chaotic map lattices defined as

$$
x_{i}^{t+1}=(1-\epsilon) f\left(x_{i}^{t}\right)+\frac{\epsilon}{2}\left(f\left(x_{i-1}^{t}\right)+f\left(x_{i+1}^{t}\right)\right) .
$$

In this equation, $t$ (resp. $i=1, \ldots, k$ ) is a temporal (resp. a spatial) index of discrete lattice, $\epsilon$ is the couple parameter, and $f$ is a logistic map. They first deal with continuous domain digitizing of all operands to be suited for 
hardware implementation. To achieve this, they consider a particular version of Equation 13 where $x$ ranges over $\left\{0,1, \ldots, 2^{k}-1\right\}$ and $f$ is a modified logistic

map, $f(x)=\left\lfloor\frac{4 x\left(2^{k}-x\right)}{2^{k}}\right\rfloor$ for a $k$-bits precision. Secondly, to avoid the finite precision chaotic map problem, they compute only the insignificant bit which is subject to be an output. Indeed, for each 25 clock cycles, only the $w=16$ most insignificant bits of the random numbers would be used from each lattice and the computational precision $k=32$.

Chaotic based Timing Reseeding (CTR) proposed first in [87] aim at removing the short period problem due to the quantization error from a nonlinear chaotic map PRNG. Instead of initializing the chaotic PRNG with a new seed, the seed can be selected by masking the current state $x^{t+1}$ at a specific time (see Figure 10). More precisely, the reseeding unit compares the two register states to check whether a fixed point has been reached. In this case $x^{t+1}$ is not streamed out. It is masked with a constant and the result is stored in the initial register state. Additionally, it increases the period each time the condition is true or the reseed period is reached (counter). This main concept of CTR was first implemented in FPGA [88], in which the Carry Lookahead Adder [89] has been used to optimise the critical path of the partial products of the multiplication operation. Unlike [88, authors of 90] present more hardware details for reducing multiplication operation resources. They also mix the output $x^{t+1}$ with an auxiliary generator $z^{t+1}$ to improve statistical tests. The mixer module is a $D X$ generator [91, whose output is as follows: $z^{t+1}=\left(z^{t}+\left(2^{28}+2^{8}\right) z^{t-7}\right)$ $\bmod \left(2^{31}-1\right)$. Then, the authors add the MSB-bit of $x^{t+1}\left(32^{t h}\right.$ bit $)$ to the 31 LSB-bits of the final output $y^{t+1}[30: 0]=x^{t+1}[30: 0] \oplus z^{t+1}[30: 0]$, which generates a full 32-bits output state and has a full period. Both uses Circular Left Shift 92] (CLS) and End-Around Carry Adder 93] (ECA) to optimize the multiplication operations. They finally suggest to choose a reseeding period that must be not only prime, but also not a multiple of the nonlinear chaotic map PRNG. The same approach has been used in 94 for plaintext encrypting/decrypting application system.

Differential Chaotic PRNG is a digitized implementation of a nonlinear 


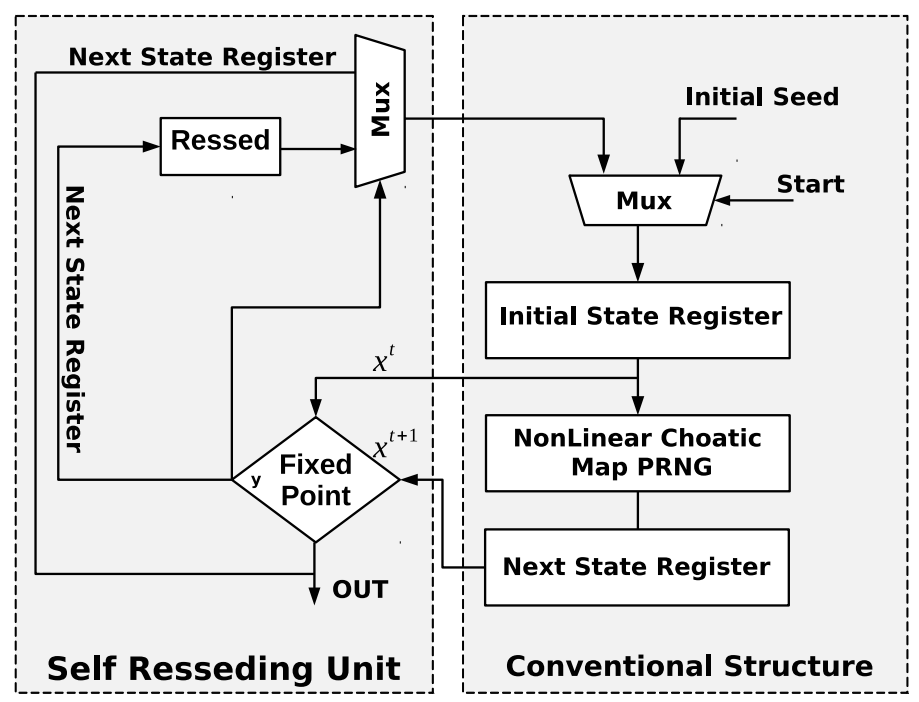

source 88

Figure 10: Chaotic based Timing Reseeding PRNG: masking the current state $x^{t+1}$ at a specific time (fixed point between the two register states is reached)

chaotic oscillator system in Rössler format 95. It uses an approximated numerical solution to solve the dynamic system generalization of the Lörenz hyperchaos. A basic representation of the dynamical system is proposed in [96, 97. Equation (Eq. (14)).

$$
\begin{gathered}
-\dddot{X}=\ddot{X}+B(\dot{X})+X \\
B(\dot{X})=\left\{\begin{array}{cc}
\alpha_{1}, & \text { if } \dot{X} \geqslant 1 \\
\alpha_{2}, & \text { otherwise, }
\end{array}\right.
\end{gathered}
$$

where, $\alpha_{1}, \alpha_{2}$ are integer values in the switch condition. The idea is to create a chaotic system with a unique equilibrium point at the origin. Indeed, to guaranties a chaotic generation, $B$ value must switch between $\alpha_{1}>1$ and $\alpha_{2}<1$.

This latter can expand in more than one direction (i.e., Euler approximation where $Y=\dot{X}$ and $Z=\ddot{X}$ ) and generates a much more complex attractor compared to other chaotic systems. 
The resolution of Equation (14) was the main study done in 98] (with other differential systems as the Chen [99] and Elwakil [96] ones). The authors deploy three different numerical methods for each system: $4^{\text {th }}$ order Runge-Kutta [100, mid-point [101, and Euler techniques [102]. Unlike the Euler techniques that only require one calculus per iteration, the mid-point provides more precise results but longer calculation paths. Additionally, the Runge-Kutta $4^{\text {th }}$-order have the longest calculation path but it has the most accurate numerical approximation. Obviously, Euler techniques show better results for implementation of differential chaotic methods in FPGA, with respects area and throughput perspectives.

More details regarding implementation and optimization of the multiplication by a constant in Equation (14) are provided in [103. In this article, authors proposed to use the Euler approximation, as illustrated in Equations (15), where the oscillation margins are within a time interval $\left[h, \alpha_{1}\right]$ ( $h$ is the Euler step).

$$
\begin{gathered}
X^{t+h}=X^{t}+h Y^{t}, \text { where } Y=\dot{X} \\
Y^{t+h}=Y^{t}+h Z^{t}, \text { where } Z=\ddot{X} \\
Z^{t+h}=Z^{t}-h\left(Z^{t}+Y^{t} B\left(Y^{t}\right)+X^{t}\right)
\end{gathered}
$$

Their optimization is based on transformation of the parameters $h=2^{-a}$ and $\alpha_{1}=2^{b}, \alpha_{2}=0$ to simplify the multiplication to a simple shift operation ( $a$ and $b$ are positive parameters). They use a Carry Lookahead Adder (CLA) 89] and a Carry Save Adder (CSA) [104 for the multiplication in the first two equations of (15), and a Carry Propagate Adder (CPA) 105] for the last one. Additionally, a post-processing is integrated for better results in statistical tests, which specifically discards the most significant bits. Authors of [79, for their part, have implemented the so-called Oscillator Frequency Dependent Negative Resistors (OFDNR) 97], which uses the same Euler approximation illustrated in Equations 15). However they have not detailed the resources they used for such multiplication on their FPGA (e.g., DSP, LUT, ...).

In 106 is presented a non-autonomous four-dimensional hyperchaotic PRNG 
based on Rössler differential equations. In such a chaotic system some undesirable behaviors can appear. Thus, an advanced process-control is necessary in order to delay the occurrence of the hyperchaos. Therefore, the authors used Euler approximation and a control function of 256 bits Linear Feedback Shift Register (LFSR), whose outputs are multiplied by the appropriate coefficient of the control function. However, a post-processing of 256-bits based Fibonacci LFSR is used to remove the short-term predictability of hyperchaotic generator and to successfully undergo the statistical tests of NIST batteries. The post processing combines two loops of rotation and XOR feedback loops. The first one uses a fixed 1-bit static rotation to suppress the short-term predictability. The second one is based on a variable rotation controlled by a Fibonacci series of $k$-bits. The differential sensitivity problem is solved by changing any bit while the other bits is propagating during $n$-cycles.

Chaotic Iteration based PRNG (CI) has been proposed in [107, 108, to implement a new post processing with the same chaos theory defined by Devaney and Li-Yorke. Chaotic iterations are defined by an initial configuration $x^{0}$, a function $f$, and a sequence $S$ said to be a chaotic strategy. At the $t$-th iteration, only the $S^{t}$-th cell is iterated. Among many proposed versions, one of them has been implemented on FPGA using BBS and XORshift PRNGs as generators. The internal state $x$ is a vector of 16-bits, whereas two 64-bits XORshift generators are provided as entropy sources. The outputs are then spread into four 32-bits integers. Then for each integer, there are 16 (2-bits) components that can be found and every 12 of these components are used to update the states. Lastly, the 4 least significant bits (LSBs) of the output BBS generator decide if the state must be updated or not with the considered 13-bits block [109, 110].

Two new families have then been proposed, which are based on chaotic iterations 111] 112] (CIPRNG-XOR) on FPGA/ASIC on the one hand, and on the deletion of an Hamilton Cycle [113] on the other hand. This latter has to satisfy some balance properties: the associated Markov chain on the $n$ 575 cube must be close enough to the uniform distribution. In these first studies, the minimum length of the chain between two uniform outputs is larger than 
109 in [114, and it is equal to 9 in [115], for a Boolean function of 8 binary variables. The new version on FPGA proposes only one jump in the $n$-cube and a permutation, as sufficient condition to pass all statistical tests in NIST and TestU01.

\section{True Random Number Generators}

We focus now on FPGA implementations of truly random number generators (TRNGs). FPGA based TRNGs are physical generators that use various hardware components of FPGAs to produce random-like numbers in a faster way than using software. These TRNGs use, as entropy source, either the electronic noise of embedded components or some environmental sensors (temperature, noise, and so on). FPGAs are thus efficient and inexpensive random number generators. Various techniques and hardware optimizations have already been proposed in the literature, while FPGA components have been used in RNG context for optimization, mixing with external components, or as post-processors.

\subsection{Phase-Locked Loop TRNGs}

The Phase-Locked Loop (PLL) 21] is a circuit derived from an external clock generator source like a quartz or a "Resistor Capacitor" circuit, which can be configured to produce a signal whose phase is associated to the phase of the input signal (see Figure 11a. This latter depends on the physical environment (power, temperature, or any other physical quantity), and it uses a jitter extraction technique as random stream, which is indeed a short-term variation of the clock propagation. Analog PLLs use the jitter caused by Voltage Controlled Oscillator (VCO) noise, while digital PLL [116] generators extract their randomness from synchronous/asynchronous Flip-Flop components. The most common jitter measurements used by FPGA vendors are, namely, the period jitter and the cycle-to-cycle one. The first jitter is defined as the difference between the $n$-th clock period and the mean clock period, while cycle-to-cycle jitter consists of the difference between adjacent clock cycles in the collection of 605 sampled clock periods. 


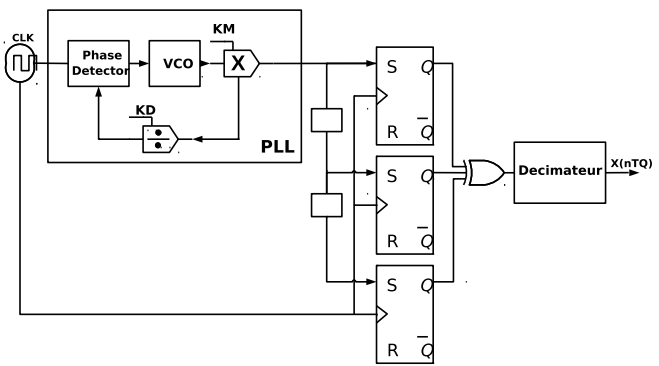

(a)PLL architecture

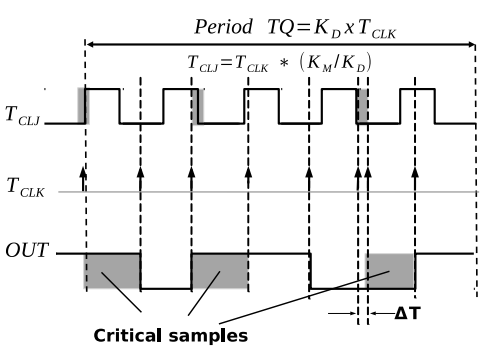

(b)PLL timing

Figure 11: Phase-Locked Loop TRNG: detecting the jitter by sampling the reference clock signal $T_{C L K}$ using a correlated signal $T_{C L J}$ synthesized in the PLL

The authors of [117] have proposed an analysis about extracting randomness from the jitter of a PLL implemented on an Altera FPGA. Their study is based on detecting the jitter by sampling the reference clock signal $T_{C L K}$ using a correlated signal $T_{C L J}$ synthesized in the PLL, where $T_{C L J}=T_{C L K} \times$ $\left(K_{M} / K_{D}\right)$ with $K_{M}$ and $K_{D}$ as PLL multiplier and divider that must be prime number constants. According to [117, the maximum distance, further denoted as $\max \left(\Delta T_{\text {min }}\right)$, between the two clocks $C L K$ and $C L J$ must satisfy $\max \left(\Delta T_{\text {min }}\right)<\sigma_{j i t}$ to be able to extract randomness. Indeed, according to the authors, in ideal environmental conditions, we have $\sigma_{j i t}=0$ (we do not have any jitter). In that situation, the sampled outputs are deterministic and can be represented by a series of a bitwise addition of $K_{D}$ input. According to these authors, the period in that situation is equal to $T_{Q}=K_{D} T_{C L K}=K_{M} T_{C L J}$. Contrarily, in real case conditions, $\sigma_{j i t}$ is necessarily negative, and so the output loses its deterministic character and becomes random. Indeed, the maximum distance $\max \left(\Delta T_{\text {min }}\right)$ between the two clocks is dependent on the jitter distribution, while the outputs has a direct impact by this latter following the expression [117:

$$
x^{t}\left(n T_{C L K}\right)=x\left(\left(n T_{C L K}\right)-\sum_{j=0}^{i} J \tau_{j}\right),
$$

where $\tau$ is the jitter and $J$ is the value of the output influenced by the jitter. The 
period is changed in $\max \left(\Delta T_{\min }\right)=T_{C L K} \times G C D\left(2 K_{M}, K_{D}\right) /\left(4 K_{M}\right)$, where $K_{D}$ is odd and $\max \left(\Delta T_{\text {min }}\right)$ is divided by 2 .

This research work has been deepened in [118 by combining more than one PLL either in parallel or in series. By doing so and due to this combination, the sensitivity $S$ to the jitter effect is significantly increased according to the formula:

$$
S=T_{C L K} \max \left(\Delta T_{\min }\right) .
$$

As expected, the lowest sensitivity is achievable by using only one PLL. In that case, the number of random samples and their entropy are low, due to a low value of $S$. To solve this problem, the authors add a second PLL, either in parallel or in a cascaded configuration, the objective being to increase the entropy without increasing too much the sensitivity.

Authors of [119] have tested the impact of "environmental" PLL conditions (encompassing its temperature, its bandwidth, etc.) on the statistical quality of the produced output. They have deduced that a low bandwidth of PLL causes a higher number of critical samples, which decreases the output jitter, and consequently increases the tracking jitter. Finally, authors in [120] propose two configurations of PLL based TRNGs in embedded systems.

\subsection{Ring Oscillator TRNGs}

A Ring Oscillator (RO) is a series of an odd number of NOT gates, whose outputs states are balanced between two voltage levels, i.e., between bit 0 an bit 1. The NOT gates, or Inverter Ring Oscillators IROs, are cascaded, while the output of the last inverter is fed back to the first inverter of that chain (see Figure 12). In [121, 122, the authors have proposed a TRNG based on two ring oscillators. This latter is rated by different clocks generated by an internal PLL implemented on FPGA. The authors have also extracted the jitter of the 2 ROs implemented in only one CLB slice.

Similarly, the authors of [123, have proposed an approach that combines ROs based on inverters with XOR gates. Their approach is close to the LFSR one, except that they use inverters, the latter being combined either using the 


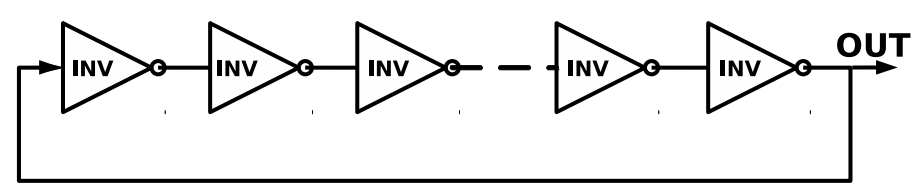

Figure 12: Inverters based ring oscillator

Fibonacci setup or the Galois one. The result also has an analog feedback to the input, where the feedback polynomial form is $f\left(x^{t}\right)=\sum_{i=0}^{k} f_{i} x^{t+i}$, with $f_{0}=f_{k}=1$. However, the inverter does no reach a fixed state if $f\left(x^{t}\right)=$

${ }_{635}\left(1+x^{t}\right) h\left(x^{t}\right)$ and the primitive polynomial is such that $h(1)=1$. Authors have finally demonstrated their ability to extract a better stable state compared to classical RO TRNG, from which randomness can be produced.

\subsection{Self-Timed Ring TRNG}

Self-Timed Ring (STR) proposed in 124, 125, 126, is an alternative approach to generate clock jitter compared to the inverter RO based TRNG. The structure of STR consists of a micropipeline architecture [127, as described in Figure 13 In this latter, a ring of $L$ stages can generate $k$-bits outputs, denoted by $y^{t}(0 \leqslant$ $k \leqslant L-1$ ), at each stage and with a propagation phase equal to $\Delta \varphi=T / 2 L$. A stage consists of a Muller gate and an inverter. Therefore the jitter period in STRs, for each ring stage, can be considered as an independent entropy source compared to the propagation of one event all around the ring in IRO.

Two situations can occur. If the outputs of two successive stages are equal $\left(y^{t}=y^{t+1}\right)$, then the clock jitter is propagated forward. Conversely, in case where $y^{t} \neq y^{t-1}$, then the jitter is propagated backward. The final output sequence $\left(y^{t}\right)_{1 \leqslant k \leqslant L-1}$ is extracted at each ring stage output using a Flip-Flop, and the result is combined according to the following XOR operation: $\psi=$ $y^{1} \oplus y^{2} \oplus \cdots \oplus y^{t+k-1}$.

\subsection{Metastability TRNG}

Metastability is a phenomenon that can occur when a signal is transferred between circuitry in unrelated or asynchronous clock domains. This short time 


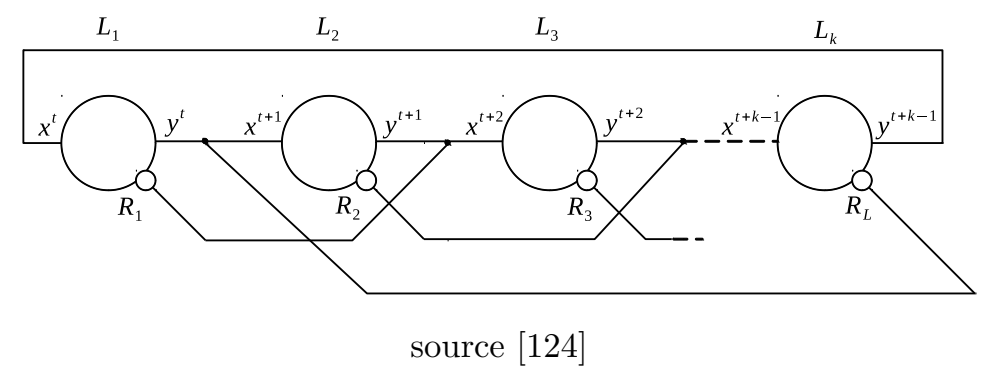

Figure 13: Self-Timed ring architecture: at each ring stage L (Muller gate and an inverter), the jitter is propagated forward if $y^{t}=y^{t+1}$ or conversely backward, when the output is the XOR of each extracted jitter by a Flip-Flop

phenomenon can cause system failures in digital devices.

Authors of [128] have presented a way to use such metastability phenomena as entropy sources generated by 5 IRO (Ring Oscillator based Inverters) stages. Their goal is to maintain metastability as long as possible, while extracting randomness from this entropy source. To achieve this objective, the authors have firstly implemented inverters as loop rings, and have used a clock generator controller module to switch the connectivity between the IRO stages following two modes, namely the metastability mode "MS" and the generation one, as described in Figure 14. By doing so, the output converges to the metastability level, and it stays a longer time in that state than when using a bi-stable circuit (Flip-Flop), causing thus a high entropy. Secondly, the authors wanted to estimate the robustness of the system after applying the sampling process in various environmental variation modes on FPGA. To achieve this second objective and for a higher quality output, they have added another stage to decrease the operation rate, by applying a Von-Neumann post-processing. Such a postprocessing stage influences the loads of the last inverter (RC parasitic). Let us finally note that, operationally speaking, the end of the IRO was implemented in ASIC while the post-processing process was achieved by using a FPGA in order to test the global device.

675 Another metastability circuit used as a TRNG has been proposed in [129]. Authors of this article have proposed to use the Flip-Flop metastability when 


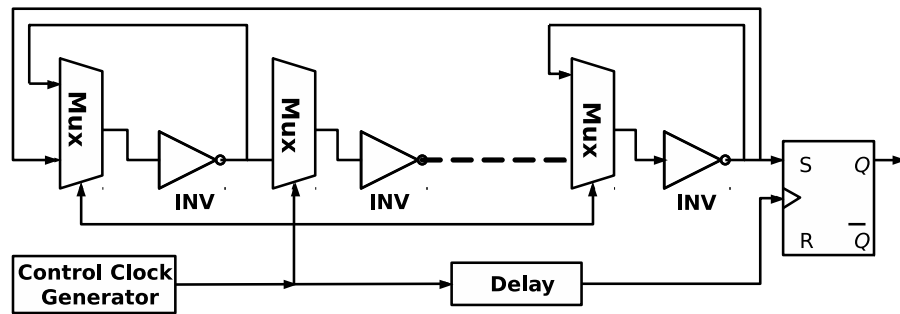

(a)Metastability based TRNG architecture

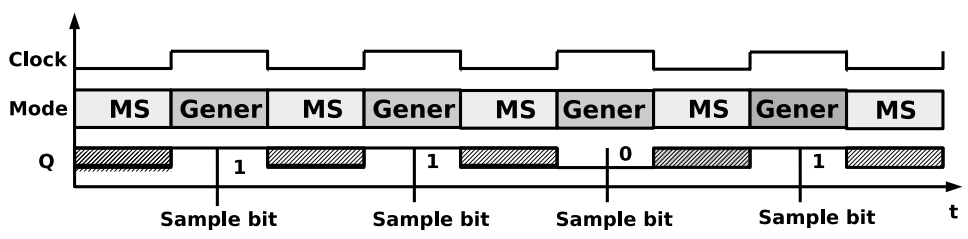

(b) Metastability timing switching based TRNG source 128

Figure 14: (a) TRNG based on the metastability of multistage architecture inverter ring oscillator, (b) The timing switching connectivity between the IRO stages following the metastability mode "MS" and the generation mode.

there is a violation in setup/hold time [130] (see Figure 15). The setup time $\mathrm{ST}$ is the amount of time a synchronous input of the Flip-Flop must be stable before the active edge of the clock. The hold time HT is the amount of time a synchronous input of the Flip-Flop must remain stable after this active edge of the clock ( $c . f$. Figure 15). The violation occurs when the input data is between these two times. However, due to their short time (ST/HT), the output must converge rapidly to a stable state 0 or 1 if the input is stable during this two times. Their system is based on a closed-loop feedback mechanism for autoadjustment on delay $\Delta$, controlled by the Programmable Delay Lines (PDLs) stage based on a LUT, in order to avoid violation and maintain metastability.

The proposed system uses at-speed monitor to keep tracking the output bit probability and the Proportional-Integral (PI) controller, in order to decide to add or subtract the delay difference. As for the updated/corrected delay difference $\Delta$, it is the difference between the bias/skew caused by the asymmetric routing $\Delta_{b}$, with delay issued by environment changes (temperature, etc.), and 


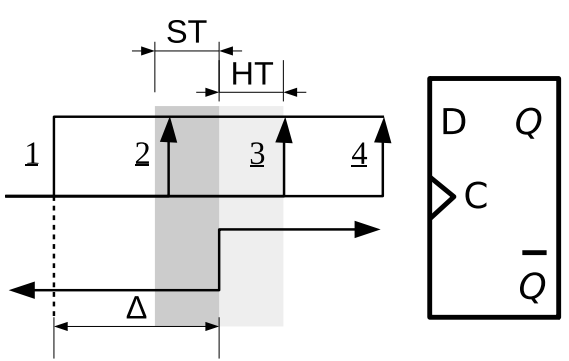

(a)Metastability creating in FF

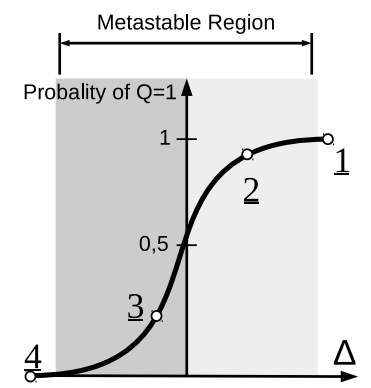

(b)Metastability timing of FF

source 131

Figure 15: (a) The setup (ST) and hold (HT) scenarios operations in Flip-Flop, (b) the output probability depending the delay difference $(\Delta)$ of the input signal

the delay $\Delta_{f}$ corresponds to the "corrected feedback delay difference" injected by PDL, according to the following formula:

$$
\Delta=\Delta_{p}+\Delta_{b}-\Delta_{f}
$$

Their method consist of tuning sampling and signal arrival times by setting $\Delta$ to 0 , which leads to the metastability of the D-Flip-Flop.

An updated version of this TRNG has finally been proposed in [131. Thanks to an analysis of probability, they reach some metastable states for a long period and prevents deterministic states. To do so, they have used an additional hardware resource as memory for storing the outputs, and a Hamming weight to calculate the history of the probability bits.

\section{Experimental Results and Hardware Analysis}

\subsection{Methodology}

Formally speaking, the space represents the allocation cost of most objects used in the algorithm (tables, indexes, loops, etc.). It can also be combination of many PRNG algorithms. In terms of FPGAs, the latter can be translated in memories, registers, and LUT resources, etc. These resources can be a sin- 
constants), algebraic functions (division, modulo, etc.), or any other elementary function. The question raised in this section is thus: how much hardware resources are needed to provide pseudorandom numbers with a good statistical profile? And which algorithms outperform the other ones in terms of internal resources, while providing higher throughput?

Almost aforementioned (P)RNGs have been evaluated regarding their hardware performance according to three parameters: (1) the area, which is the result of $(L U T+F F) \times 8$, (2) the throughput being the frequency (clock-tosetup) multiplied by the RNG output length for one clock cycle, and (3) the ratio between throughput over area in Mega bits per area unit.

\subsection{Hardware Comparison}

Hardware implementation resources required by linear (P)RNGs, their throughput, and the rate area over throughput are presented in Figure 16, when nonlinear ones are in Figure 17. Finally, the TRNGs are represented in Figure 18.

Let us start start to discuss the results obtained with linear PRNGs, as illustrated in Figure 16. It appears clearly that the cellular automata has the lowest area, when compared to the other approaches. Such results can be explained by the need of a low amount of resources to store both the states and the rules in the cellular automata. Conversely, the TGFSR family deploys BRAM block memories to read 3 word and write the output in one cycle, whereas LFSR family uses more LUTs in order to parallelize the shifting process based on the polynomial equation. Another parameter is the use of black box as DSP and block memories. The latter optimize the logic operation as multiplication, support the floating point, store internal process in a multidimensional bloc, and finally read and write multiple states in parallel from the BRAM. These advantages, leading to the difficulty to compare such designs to other ones that do not have that, lead naturally to further area bloc consumption in the case of an ASIC implementation. As a consequence, we will consider that (P)RNGs without black boxes are better and more recommended for cryptographic applications.

In terms of area, the PRNGs based on cellular automata 62, 64, 67] have 
the lowest resource occupation of FPGA, if we compare them to the other ones (see Figure 16a). By comparison, the PRNG based on LFSR [41] is 76 times larger. We can also remark that most TGFSR implementations do not consider the seed process, while its computing increases the area and decreases the throughput, during the load of 632 words sequentially in the block memories. The throughput, for its part, is completely related to both data path and width (dynamic range) of the design. Additionally, we must take under consideration the fact that most linear PRNGs are 32 bits ones, while the throughput increases with generators manipulating more than 64 bits. However, as stated previously, disabling DSPs and Block memories induces a decrease in the frequency and the throughput respectively. Figure $16 \mathrm{~b}$ illustrates opposed results for the area, where the LFSR based LUT family has the largest throughput of $343 \mathrm{Gbps}$, while it is 5 Gbps for the Mersenne Twister. However, the latter are for 1,042 bits and 128 bits respectively, when for 32 bits, we have 128 Gbps for the LFSRLUT [48.

Let us focus now on the Throughput/Area ratio (see Figure 16c). Here, the LUT and shift register based design [48, outperforms all the other linear PRNGs: the ratio is twice as efficient as the second best one [54, which is the Mersenne Twister based PRNG with parallel BRAM.

750 The performance of PRNGs belonging in the chaotic category are illustrated in Figure 17. The one that is based on the logistic map has the lowest area occupation. However, some differential chaotic PRNGs can be presented as good competitors, namely the chaotic iterations based PRNGs. Results obtained concerning area (Figure 17a) can be explained by the use of a basic operation (the shift one), and because the bionic coefficient $\alpha$ can be considered as a constant when implementing the logistic map. PRNGs based on chaotic iterations, for their part, need to embed linear PRNGs for their strategies: on the one hand, CIPRNG-XOR uses 3 PRNGs, while on the other hand ICGPRNG manipulates only one, but with a permutation function. Figure 17billustrates the superiority of chaotic iterations family, in which the differential PRNG based Euler optimization [103, an optimized logistic map 82, and these PRNG based chaotic 
iterations 113 , have the largest throughput in this category of chaotic generators. Regarding the Throughput/Area ratio in Figure $17 \mathrm{c}$, the chaotic PRNG based on LCGM [79] outperforms all the other linear PRNGs: the ratio is 4.1 times more efficient than the second best one [107, which is a chaotic iterations generator based on BBS and XORshift.

Finally, considering the TRNG analysis, only a throughput comparison is provided in Figure 18. Indeed, all the considered authors prefer not to discuss about area... which is so low when compared with PRNGs. Hence, even with this main advantage of optimized resources usage, the throughput is too low and it ranges from $\mathrm{Hz}$ to just a few $k H z$. Compared to PRNGs, TRNGs are probably more secure, while PRNGs can be deploied as fast generators.

As a conclusion, linear PRNGs can play an important role for FPGA applications, due to their rapidity and parallel generation, if we compare them to other pseudorandom generators. Chaotic PRNGs, for their part, are more secure. They are non linear PRNGs and have low hardware resources compared to the linear ones. Finally, despite the low throughput generated by the TRNG, they are still consuming only a few logic while generating a real random output.

\section{Statistical Test Analysis}

Statistical tests are used to evaluate whether the output of a given RNG can be separated from a real random sequence obtained, for instance, by rolling a dice. Such tests are usually grouped in "Batteries", like the FIPS [132, DieHARD [25], NIST SP800 - 22 [133, TestU01 [26], or AIS [134] ones. In what follows, the content of these tests is recalled, for completeness purpose so as to make our article self-contained.

The National Institute of Standard and Technologies introduced their first test battery namely Federal Information Processing Standard (FIPS) 140-1 [132] in 1994. These quick result tests have been further updated to the FIPS 1402 [135] version, which covers more complex test batteries (focused for instance on security level). 


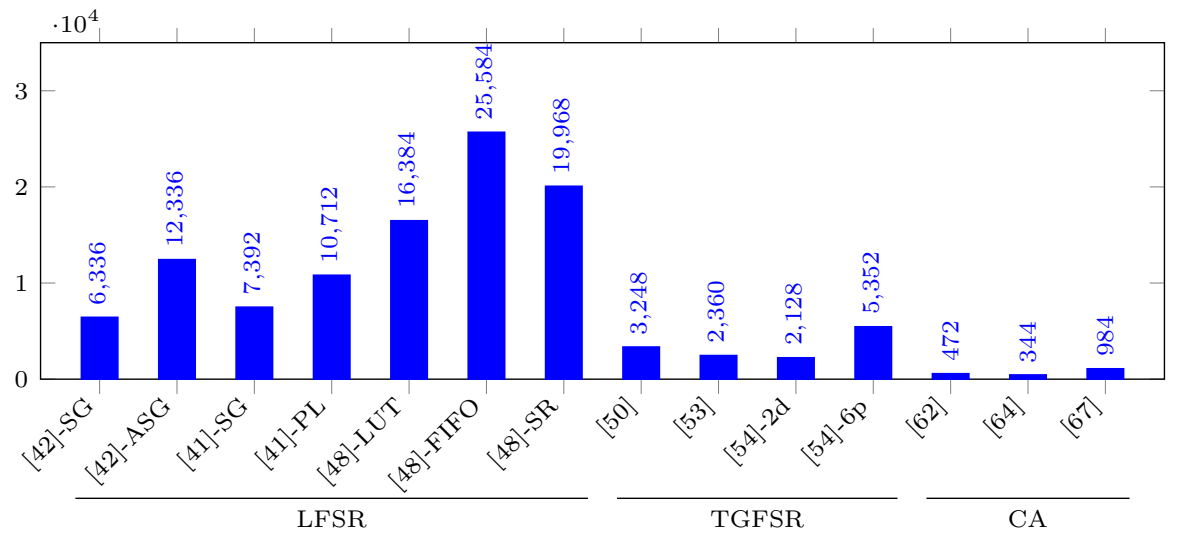

(a)Area $((\mathrm{LUT}+\mathrm{FF}) \times 8)$

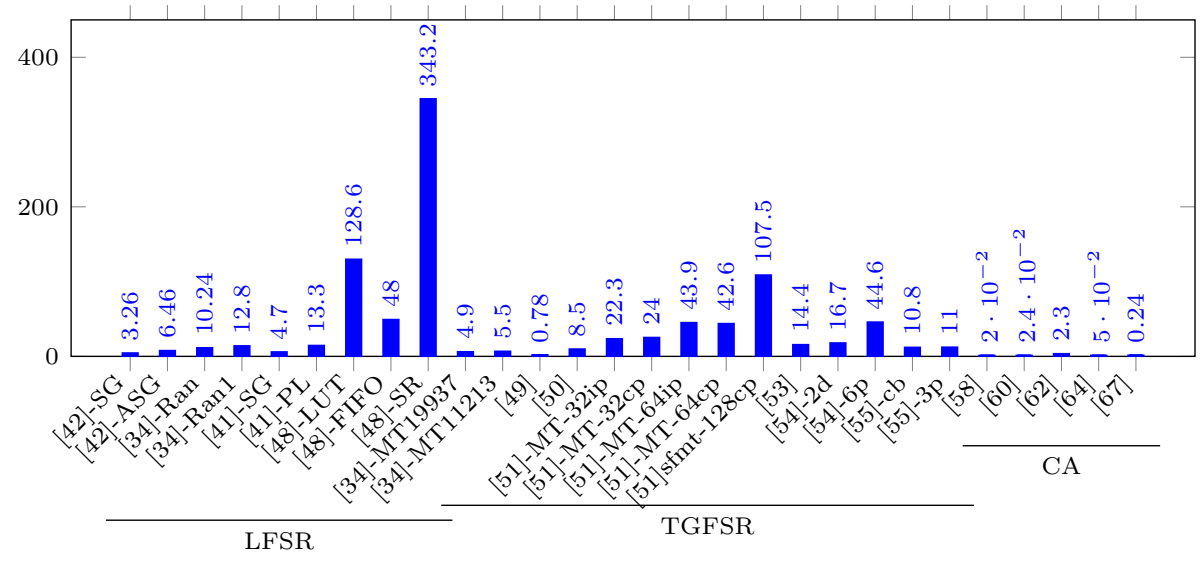

(b)Throughput (Gbps)

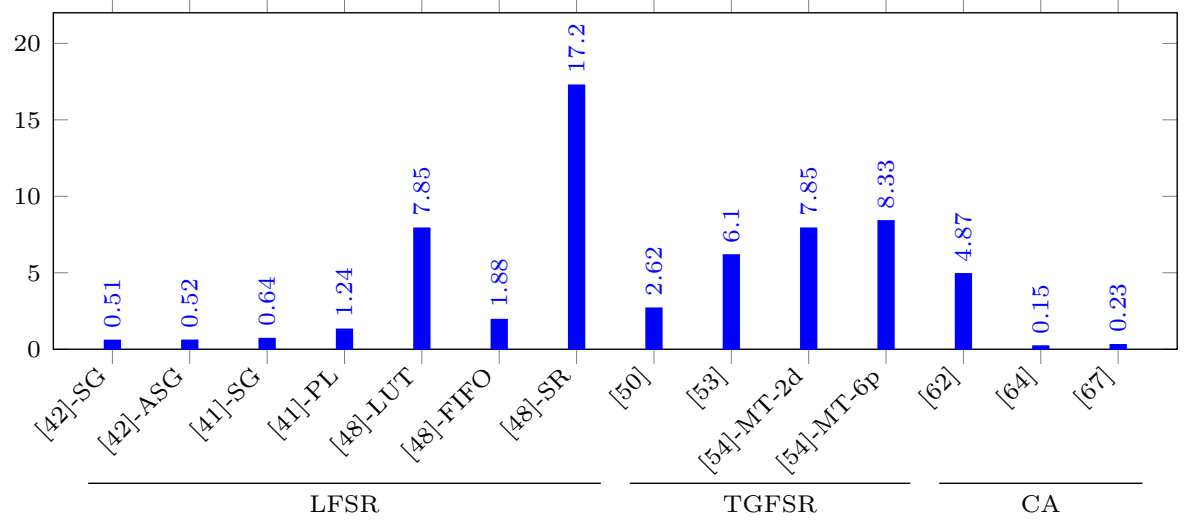

(c)Ratio (Throughput/Area) (Mbit per area)

Figure 16: Linear PRNGs FPGA hardware analysis. 


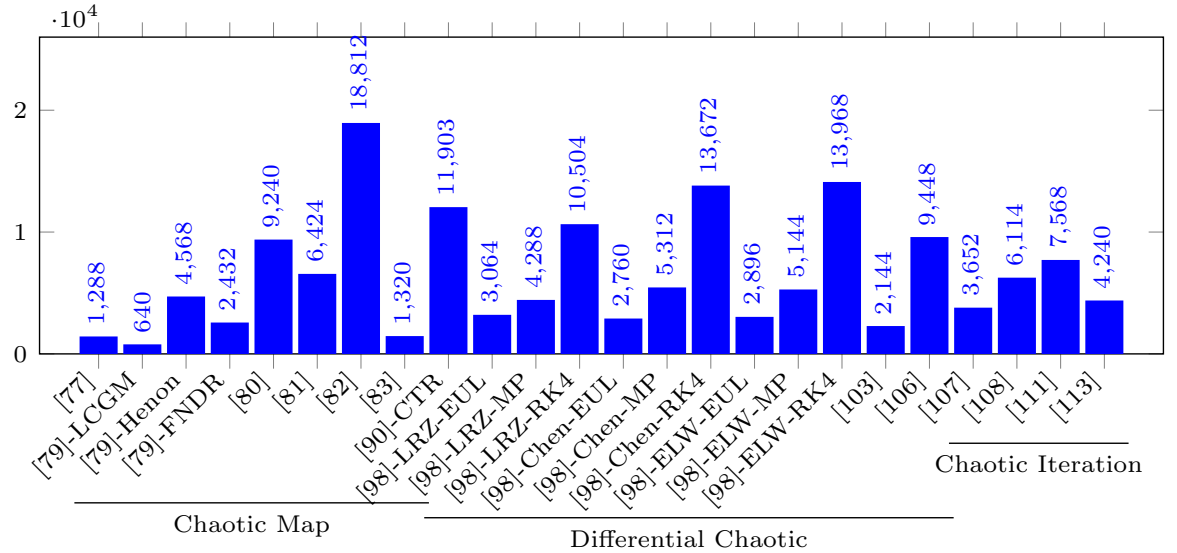

(a)Area $((\mathrm{LUT}+\mathrm{FF}) \times 8)$

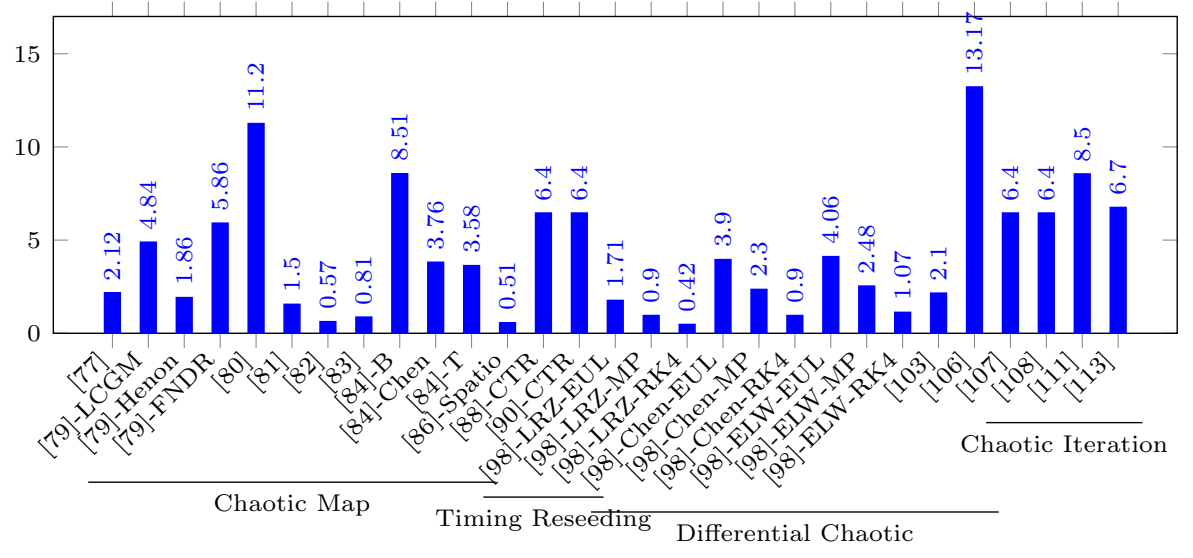

(b)Throughput (Gbps)

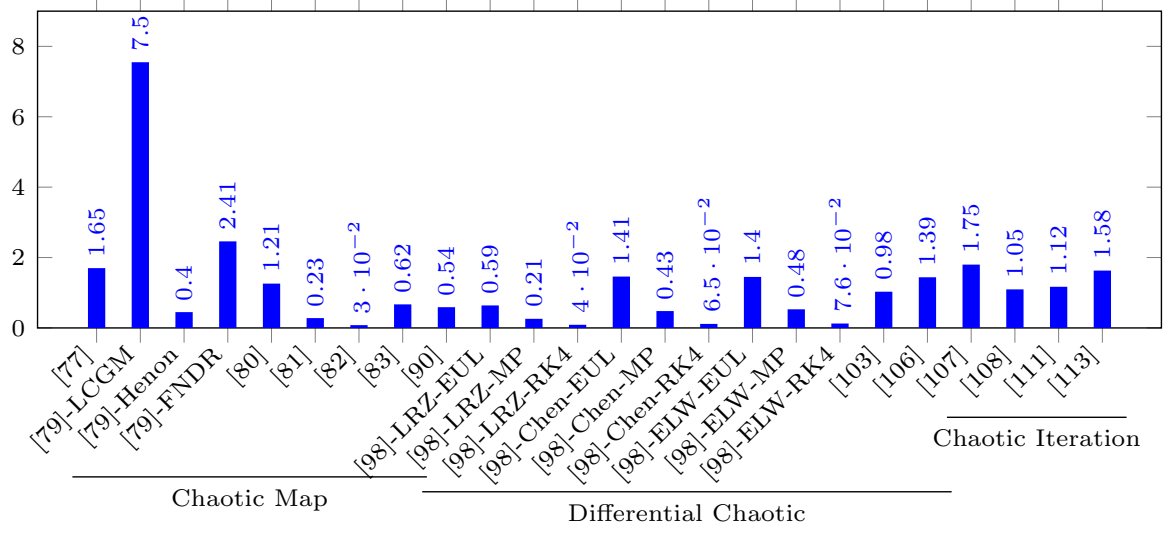

(c)Ratio (Throughput/Area) (Mbit per area)

Figure 17: Non-lineair PRNGs FPGA hardware analysis. 


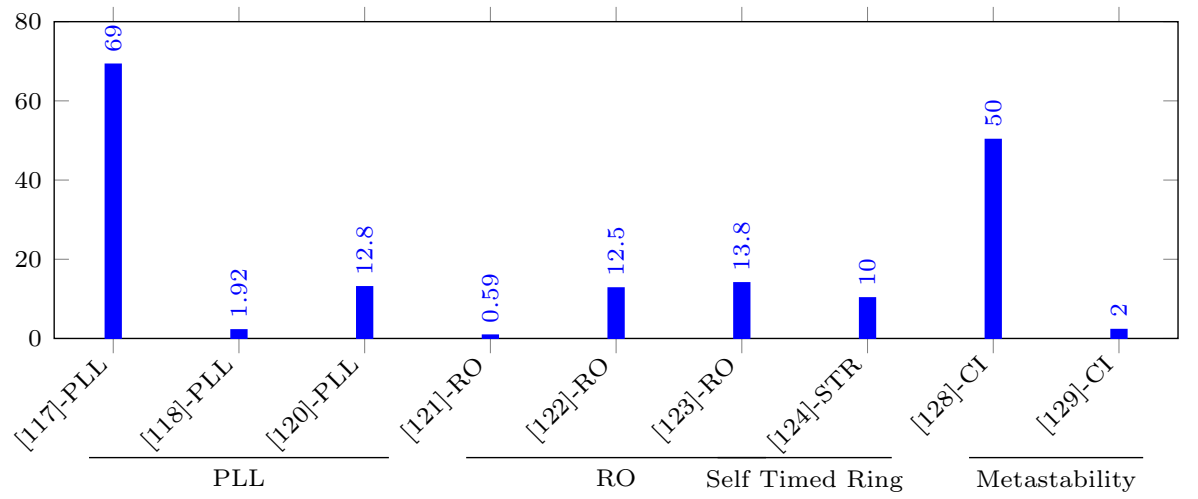

Figure 18: TRNGs FPGA implementation analysis: Throughput (Mbps).

Meanwhile, the DieHARD battery has been proposed by George Marsaglia [25]. It contains 18 tests of randomness. It was designed to provide a better way of analysis in comparison to the previously released NIST tests. Unlike this latter, the $p$-values have now to belong to some fixed chosen interval $[\alpha, 1-\alpha]$, with a signification level of $\alpha$ for $5 \%$ for instance. An example of these batteries are: "Birthday spacings", "Overlapping permutations", "Ranks of matrices", "Monkey tests", "Count the 1's", "Parking lot", "Minimum distance", "Random spheres", "The sqeeze", "Overlapping sums", "Runs", and "The craps".

The AIS-31 battery [134 is a German standard to test and evaluate the security properties of truly random number generators. It uses 9 statistical tests for the evaluation of a TRNG. AIS can be divided in two categories: the first one consists of T0-T4, which are the same function of FIPS 140-1 [132]. These later are mostly used to test the outputs of a post-processing. T0 is the "disjointedness test", which collects 65536 of 48-bit and verifies that two adjacent values must not be equal. T1 is the monobit test, $\mathrm{T} 2$ is the poker test, T3 is the run test, and T4 is the longest run test. As for T5, it is part, is the auto-correlation test, and T6 is a "uniform distribution test" including of 2 sub-tests. T7 is a "comparative test for multinomial distributions", and finally T8 is an entropy test (Coron's test).

In the other side, National Institute of Standard and Technologies introduces 
a new test battery known as "NIST SP800 - 22" [133. This one aims at testing the random profile of a given sequence using 15 tests. More precisely, it evaluates a long binary sequences generated by the RNG for the randomness and a higher security testing level than the FIPS 140-2. The tested sequences must have a fixed length $N$, where the parameter $N$ is such that $10^{3}<N<10^{7}$. Then, for each statistical test, a set of $s$ sequences is produced by the RNG under test, and $p$-values are obtained. They all need to be larger than 0.0001 to reasonably consider the associated sequences as uniformly distributed and cryptographically secure according to NIST standards.

The TestU01 battery is now the most complete and stringent battery of tests for RNG [26]. It was initially developed by "Pierre L'Ecuyer" and was implemented in the ANSI C language with more than 516 tests grouped inside 7 big sub-batteries. This new battery of tests covers various classical tests already present in other batteries with new algorithms for performance and cryptographic tests.

\subsection{Statistical results of FPGA based $R N G$}

In Table 1 and 2, a number of generators are classified according to the battery test they have undergone. As it can be observed, the most stringent battery (Big crush) has only been applied twice in the literature, namely [107, 122]. Let us notice that most (P)RNGs pass the Diehard and NIST batteries, while only a few PRNGs have been tested using the FIPS that has been integrated latter inside the NIST. Considering the TestU01 one, only crush batteries are usually considered. All generators fail at least one test, with the exception of chaotic iterations generators that can pass the whole battery.

Authors in [111, 112] investigate the related problem for linear PRNGs. They show too that usual chaotic PRNGs are not passing the BigCrush when they consider its non linearity. However, being linear does not lead to a high linear complexity, which is defined by the degree of their polynomial characteristic function. However, most random number generators are linear recursive, and so 
test characterizes the $(\mathrm{P}) \mathrm{RNG}$ by their longest LFSR model: non randomness is claimed when the model is too short. This model is estimated by using the well-known Berlekamp-Massey algorithm [136]. It determines the shortest polynomial of a linearly recurrent finite output sequence in $\mathrm{GF}_{2}$. Note that all the other generators fail too the linear complexity test, except for PCG32 and MRG32K3a: indeed, only PRNGs based on chaotic iterations are passing TestU01. Under this category, the authors propose too an extended internal space of 64 bits (CIPRNG-XOR) for 32 bits generators, when they increase the number of internal iterations to be uniformly distributed and to pass statistical tests.

Finally, TRNGs are hard to test with TestU01 (specially the BigCrush battery), as it needs $10^{38}$ random bits for a full test. Figure 18 shows a general throughput of the order of Kbps, which makes it difficult to collect the minimum amount of data needed in such tests. Under these conditions, only the TRNG of [122] based on ring oscillators has been proven to pass with success the BigCrush battery. Note finally that other batteries offer more flexibility and need a lower amount of bits for their embedded tests (namely, Diehard, NIST, and AIS), but they are less stringent and trustworthy than TestU01.

\section{Conclusion}

We have provided a widespread coverage of the current research in hardware implementation of random number generators on FPGA. We have first recalled well known "linear generators", encompassing LCGs, LFSRs, look-up table optimised ones, twisted generalized feedback shift registers, and cellular automata. We next have deeply investigated the non-linear ones, based on Blum-BlumShub or on chaotic maps. Then a large review of the true random number generators for FPGA has been proposed, encompassing respectively the phaselocked loop, the ring oscillator, the self-timed ring, and the stability TRNG. For each type of RNG, a hardware analysis regarding area and throughput has been provided. A section about statistical tests has finally been proposed, contain- 
Table 1: Statistical Tests Analysis: Diehard, FIPS, and NIST

\begin{tabular}{|c|c|c|c|}
\hline RNG & Diehard & FIPS & NIST \\
\hline PRNG & $\begin{array}{c}{[137]} \\
{[46]} \\
62] \\
{[54,53]} \\
{[51,34} \\
662,64] \\
67,65]\end{array}$ & $\begin{array}{l}86 \\
88 \\
8\end{array}$ & $\begin{array}{c}{[42]} \\
41] \\
43,72] \\
{[103,108]} \\
{[86,106]} \\
67,60] \\
\text { Li and Chen } 88,90 \\
\text { 138. }\end{array}$ \\
\hline TRNG & $\begin{array}{l}{[122} \\
{[124}\end{array}$ & $\begin{array}{c}{[119]} \\
\text { Cherkaoui }[124,126] \\
{[128]}\end{array}$ & $\begin{array}{c}117] \\
118] \\
121] \\
120] \\
\text { Cherkaoui }[124,126 \\
{[129,131]}\end{array}$ \\
\hline
\end{tabular}

ing the detail of state-of-the-art batteries of tests, and the test results of some generators reviewed in this article against these batteries.

[1] D. E. Knuth, The Art of Computer Programming, Volume 2 (3rd Ed.): Seminumerical Algorithms, Addison-Wesley Longman Publishing Co., Inc., Boston, MA, USA, 1997.

[2] J. E. Gentle, Random number generation and Monte Carlo methods, Springer Science \& Business Media, 2003.

[3] C.-E. Froberg, C. E. Frhoberg, Introduction to numerical analysis, Addison-Wesley Reading, Massachusetts, 1969.

[4] M. G. Luby, Pseudorandomness and cryptographic applications, Princeton University Press, 1996. 
Table 2: Statistical Tests Analysis: TestU01 Crush and BigCrush, AIS

\begin{tabular}{|c|c|c|c|}
\hline RNG & $\begin{array}{l}\text { TestU01 } \\
\text { Crush }\end{array}$ & $\begin{array}{l}\text { TestU01 } \\
\text { BigCrush }\end{array}$ & AIS \\
\hline PRNG & $\begin{array}{c}137 \\
\text { Thomas } 46,47,48] \\
{[54,53} \\
51 \\
139] \\
77,80 \\
83\end{array}$ & [107] 111] 113 & \\
\hline TRNG & & 122 & $\begin{array}{c}\text { Cherkaoui }[124,126] \\
{[128}\end{array}$ \\
\hline
\end{tabular}

[5] S. Even, Y. Mansour, A construction of a cipher from a single pseudorandom permutation, Journal of Cryptology 10 (3) (1997) 151-161.

[6] T. ElGamal, A public key cryptosystem and a signature scheme based on discrete logarithms, in: Advances in Cryptology, Springer, 1985, pp. $10-18$.

[7] M. Henson, S. Taylor, Memory encryption: a survey of existing techniques, ACM Computing Surveys (CSUR) 46 (4) (2014) 53.

[8] L. Lamport, Constructing digital signatures from a one-way function, Tech. rep., Technical Report CSL-98, SRI International Palo Alto (1979).

[9] C. Shannon, Communication theory of secrecy systems, Bell System Technical Journal, The 28 (4) (1949) 656-715.

[10] L. Tippett, Random Sampling Numbers. Arranged by L.H.C. Tippett, Etc, [Tracts for Computers. no. 15.], 1927.

URL http://books . google.dz/books?id=CZJTMwEACAAJ 
[11] M. Campbell-Kelly, M. Croarken, R. Flood, E. Robson, The history of mathematical tables, AMC 10 (2005) 12.

[12] M. G. Kendall, B. B. Smith, Randomness and random sampling numbers, Journal of the royal Statistical Society (1938) 147-166.

[13] S. H. Lavington, A history of Manchester computers, NCC Publications, 1975.

[14] G. W. Brown, History of rand's random digits, summary, Tech. rep., DTIC Document (1949).

[15] W. Thomson, Ernie-a mathematical and statistical analysis, Journal of the Royal Statistical Society. Series A (General) (1959) 301-333.

[16] N. Metropolis, The beginning of the monte carlo method, Los Alamos Science 15 (584) (1987) 125-130.

[17] H. Niederreiter, N.-C. R. C. on Random Number Generation, Random number generation and quasi-Monte Carlo methods, Vol. 63, SIAM, 1992.

[18] P. L'Ecuyer, Uniform random number generation, Annals of Operations

[19] C. D. Motchenbacher, J. A. Connelly, Low-noise electronic system design, Wiley New York, 1993.

[20] L. Kleeman, A. Cantoni, Metastable behavior in digital systems, Design \& Test of Computers, IEEE 4 (6) (1987) 4-19.

915

[21] G.-C. Hsieh, J. C. Hung, Phase-locked loop techniques. a survey, Industrial Electronics, IEEE Transactions on 43 (6) (1996) 609-615.

[22] R. H. Freeman, H.-C. Hsieh, Distributed memory architecture for a configurable logic array and method for using distributed memory, uS Patent 5,343,406 (Aug. 30 1994). 
[23] P. M. Freidin, Logic block with look-up table for configuration and memory, uS Patent 5,414,377 (May 9 1995).

[24] E. Barker, A. Roginsky, Draft NIST special publication 800-131 recommendation for the transitioning of cryptographic algorithms and key sizes (2010).

[25] G. Marsaglia, The diehard test suite, 1995, URL http://stat. fsu. edu/ geo/diehard. html.

[26] P. L'Ecuyer, R. Simard, Testu01: Ac library for empirical testing of random number generators, ACM Transactions on Mathematical Software (TOMS) 33 (4) (2007) 22.

[27] P. L'Ecuyer, F. Panneton, Fast random number generators based on linear recurrences modulo 2: overview and comparison, in: Proceedings of the Winter Simulation Conference, 2005., 2005, pp. 10 pp.--

[28] M. Matsumoto, T. Nishimura, Mersenne twister: a 623-dimensionally equidistributed uniform pseudo-random number generator, ACM Transactions on Modeling and Computer Simulation (TOMACS) 8 (1) (1998) $3-30$.

[29] R. C. Tausworthe, Random numbers generated by linear recurrence modulo two, Mathematics of Computation 19 (90) (1965) 201-209.

[30] D. E. Knuth, Deciphering a linear congruential encryption, IEEE Transactions on Information Theory 31 (1) (1985) 49-52.

[31] T. G. Lewis, W. H. Payne, Generalized feedback shift register pseudorandom number algorithm, Journal of the ACM (JACM) 20 (3) (1973) $456-468$.

[32] M. Matsumoto, Y. Kurita, Twisted gfsr generators, ACM Transactions on Modeling and Computer Simulation (TOMACS) 2 (3) (1992) 179-194. 
[33] G. S. Fishman, L. R. Moore, III, An exhaustive analysis of multiplicative congruential random number generators with modulus $2 \wedge 31-1$, SIAM Journal on Scientific and Statistical Computing 7 (1) (1986) 24-45.

[34] S. Banks, P. Beadling, A. Ferencz, Fpga implementation of pseudo random number generators for monte carlo methods in quantitative finance, in: Reconfigurable Computing and FPGAs, 2008. ReConFig'08. International Conference on, IEEE, 2008, pp. 271-276.

[35] W. H. Press, Numerical recipes 3rd edition: The art of scientific computing, Cambridge university press, 2007.

955 [36] G. Marsaglia, et al., Xorshift rngs, Journal of Statistical Software 8 (14) (2003) 1-6.

[37] G. Marsaglia, A. Zaman, A new class of random number generators, The Annals of Applied Probability (1991) 462-480.

[38] A. K. Oudjida, N. Chaillet, Radix- $2^{r}$ arithmetic for multiplication by a constant, IEEE Transactions on Circuits and Systems II: Express Briefs 61 (5) (2014) 349-353.

[39] A. K. Oudjida, A. Liacha, M. Bakiri, N. Chaillet, Multiple constant multiplication algorithm for high-speed and low-power design, IEEE Transactions on Circuits and Systems II: Express Briefs 63 (2) (2016) 176-180.

${ }_{965}$ [40] A. K. Oudjida, N. Chaillet, M. L. Berrandjia, Radix-2r arithmetic for multiplication by a constant: Further results and improvements, IEEE Transactions on Circuits and Systems II: Express Briefs 62 (4) (2015) $372-376$.

[41] R. S. Katti, S. K. Srinivasan, Efficient hardware implementation of a new 970 pseudo-random bit sequence generator, in: Circuits and Systems, 2009. ISCAS 2009. IEEE International Symposium on, IEEE, 2009, pp. 13931396. 
[42] E. Erkek, T. Tuncer, The implementation of asg and sg random number generators, in: System Science and Engineering (ICSSE), 2013 International Conference on, 2013, pp. 363-367.

[43] V. R. Gonzalez-Diaz, F. Pareschi, G. Setti, F. Maloberti, A pseudorandom number generator based on time-variant recursion of accumulators, Circuits and Systems II: Express Briefs, IEEE Transactions on 58 (9) (2011) $580-584$.

[44] V. Friedman, The structure of the limit cycles in sigma delta modulation, Communications, IEEE Transactions on 36 (8) (1988) 972-979.

[45] F. Maloberti, E. Bonizzoni, A. Surano, Time variant digital sigma-delta modulator for fractional-n frequency synthesizers, in: Radio-Frequency Integration Technology, 2009. RFIT 2009. IEEE International Symposium on, IEEE, 2009, pp. 111-114.

[46] D. B. Thomas, W. Luk, Fpga-optimised high-quality uniform random number generators, in: Proceedings of the 16th international ACM/SIGDA symposium on Field programmable gate arrays, ACM, 2008, pp. 235-244.

[47] D. B. Thomas, W. Luk, Fpga-optimised uniform random number generators using luts and shift registers, in: Field Programmable Logic and Applications (FPL), 2010 International Conference on, IEEE, 2010, pp. $77-82$.

[48] D. B. Thomas, W. Luk, The lut-sr family of uniform random number generators for fpga architectures, Very Large Scale Integration (VLSI) Systems, IEEE Transactions on 21 (4) (2013) 761-770.

[49] S. Chandrasekaran, A. Amira, High performance fpga implementation of the mersenne twister, in: Electronic Design, Test and Applications, 2008. DELTA 2008. 4th IEEE International Symposium on, IEEE, 2008, pp. $482-485$. 
[50] X. Tian, K. Benkrid, Mersenne twister random number generation on fpga, cpu and gpu, in: Adaptive Hardware and Systems, 2009. AHS 2009. NASA/ESA Conference on, IEEE, 2009, pp. 460-464.

[51] I. L. Dalal, J. Harwayne-Gidansky, D. Stefan, On the fast generation of long-period pseudorandom number sequences, in: Systems, Applications and Technology Conference, 2008 IEEE Long Island, IEEE, 2008, pp. 1-9.

[52] M. Saito, M. Matsumoto, Simd-oriented fast mersenne twister: a 128bit pseudorandom number generator, in: Monte Carlo and Quasi-Monte Carlo Methods 2006, Springer, 2008, pp. 607-622.

[53] Y. Li, J. Jiang, H. Cheng, M. Zhang, S. Wei, An efficient hardware random number generator based on the mt method, in: Computer and Information Technology (CIT), 2012 IEEE 12th International Conference on, IEEE, 2012, pp. 1011-1015.

[54] S. Wu, J. Jiang, Y. Fu, Hardware architecture for the parallel generation of long-period random numbers using mt method, in: Computer Engineering and Technology, Springer, 2013, pp. 8-15.

[55] P. Echeverría, M. López-Vallejo, High performance fpga-oriented mersenne twister uniform random number generator, Journal of Signal Processing Systems 71 (2) (2013) 105-109.

[56] J. Von Neumann, A. W. Burks, et al., Theory of self-reproducing automata, IEEE Transactions on Neural Networks 5 (1) (1966) 3-14.

[57] J. Gleick, Chaos: Making a new science, Random House, 1997.

[58] P. Anghelescu, E. Sofron, S. Ionita, Vlsi implementation of high-speed cellular automata encryption algorithm, in: Semiconductor Conference, 2007. CAS 2007. International, Vol. 2, IEEE, 2007, pp. 509-512.

[59] I. Dogaru, R. Dogaru, Algebraic normal form for rapid prototyping of elementary hybrid cellular automata in fpga, in: Electrical and Electronics 
Engineering (ISEEE), 2010 3rd International Symposium on, IEEE, 2010, pp. $277-280$.

[60] D. Ioana, D. Radu, Fpga implementation and evaluation of two cryptographically secure hybrid cellular automata, in: Communications (COMM), 2014 10th International Conference on, IEEE, 2014, pp. 1-4.

[61] T. E. Tkacik, A hardware random number generator, in: Cryptographic Hardware and Embedded Systems-CHES 2002, Springer, 2003, pp. 450453.

[62] J. C. Cerda, C. D. Martinez, J. M. Comer, D. H. Hoe, An efficient fpga random number generator using lfsrs and cellular automata, in: Circuits and Systems (MWSCAS), 2012 IEEE 55th International Midwest Symposium on, IEEE, 2012, pp. 912-915.

[63] S.-U. Guan, S. K. Tan, Pseudorandom number generator-the self programmable cellular automata, in: Knowledge-Based Intelligent Information and Engineering Systems, Springer, 2003, pp. 1230-1235.

[64] J. M. Comer, J. C. Cerda, C. D. Martinez, D. H. Hoe, Random number generators using cellular automata implemented on fpgas, in: System Theory (SSST), 2012 44th Southeastern Symposium on, IEEE, 2012, pp. $67-72$.

[65] L. Raut, D. H. Hoe, Stream cipher design using cellular automata implemented on fpgas, in: System Theory (SSST), 2013 45th Southeastern Symposium on, IEEE, 2013, pp. 146-149.

[66] M. David, D. C. Ranasinghe, T. Larsen, A2u2: a stream cipher for printed electronics rfid tags, in: RFID (RFID), 2011 IEEE International Conference on, IEEE, 2011, pp. 176-183.

[67] L. Kotoulas, D. Tsarouchis, G. C. Sirakoulis, I. Andreadis, 1-d cellular automaton for pseudorandom number generation and its reconfigurable 
hardware implementation, in: Circuits and Systems, 2006. ISCAS 2006.

Proceedings. 2006 IEEE International Symposium on, IEEE, 2006, pp. $4-\mathrm{pp}$.

[68] L. Blum, M. Blum, M. Shub, A simple unpredictable pseudo-random number generator, SIAM Journal on computing 15 (2) (1986) 364-383.

[69] K. Sewak, P. Rajput, A. K. Panda, Fpga implementation of 16 bit bbs and lfsr pn sequence generator: A comparative study, in: Electrical, Electronics and Computer Science (SCEECS), 2012 IEEE Students' Conference on, IEEE, 2012, pp. 1-3.

[70] P. Peris-Lopez, E. San Millan, J. C. van der Lubbe, L. A. Entrena, Cryptographically secure pseudo-random bit generator for rfid tags, in: Internet Technology and Secured Transactions (ICITST), 2010 International Conference for, IEEE, 2010, pp. 1-6.

[71] P. L. Montgomery, Modular multiplication without trial division, Mathematics of computation 44 (170) (1985) 519-521.

${ }_{1070}[72]$ K. H. Tsoi, K. Leung, P. H. W. Leong, Compact fpga-based true and pseudo random number generators, in: Field-Programmable Custom Computing Machines, 2003. FCCM 2003. 11th Annual IEEE Symposium on, IEEE, 2003, pp. 51-61.

[73] L. M. Pecora, T. L. Carroll, Synchronization in chaotic systems, Physical review letters 64 (8) (1990) 821.

[74] S. Wiggins, Introduction to applied nonlinear dynamical systems and chaos, Vol. 2, Springer Science \& Business Media, 2003.

[75] R. M. May, et al., Simple mathematical models with very complicated dynamics, Nature 261 (5560) (1976) 459-467.

${ }_{1080}$ [76] M. Hénon, A two-dimensional mapping with a strange attractor, Communications in Mathematical Physics 50 (1) (1976) 69-77. 
[77] P. Dabal, R. Pelka, A chaos-based pseudo-random bit generator implemented in fpga device, in: Design and Diagnostics of Electronic Circuits \& Systems (DDECS), 2011 IEEE 14th International Symposium on, IEEE, 2011, pp. 151-154.

[78] W. Padgett, D. Anderson, Fixed-Point Signal Processing, Synthesis lectures on signal processing, Morgan \& Claypool, 2009.

URL http://books .google.dz/books?id=h590cd_BagMC

[79] P. Dabal, R. Pelka, Fpga implementation of chaotic pseudo-random bit generators, in: Mixed Design of Integrated Circuits and Systems (MIXDES), 2012 Proceedings of the 19th International Conference, IEEE, 2012, pp. 260-264.

[80] P. Dabal, R. Pelka, A study on fast pipelined pseudo-random number generator based on chaotic logistic map, in: Design and Diagnostics of Electronic Circuits Systems, 17th International Symposium on, 2014, pp. 195-200.

[81] A. Pande, J. Zambreno, Design and hardware implementation of a chaotic encryption scheme for real-time embedded systems, in: Signal Processing and Communications (SPCOM), 2010 International Conference on, IEEE, 2010, pp. $1-5$.

[82] S. Liu, J. Sun, Z. Xu, Z. Cai, An improved chaos-based stream cipher algorithm and its vlsi implementation, in: Networked Computing and Advanced Information Management, 2008. NCM'08. Fourth International Conference on, Vol. 2, IEEE, 2008, pp. 191-197.

1105 [83] L. Merah, A. ALI-PACHA, N. H. SAID, Coupling two chaotic systems in order to increasing the security of a communication system-study and real time fpga implementation.

[84] P. Giard, G. Kaddoum, F. Gagnon, C. Thibeault, Fpga implementation and evaluation of discrete-time chaotic generators circuits, in: IECON 
2012-38th Annual Conference on IEEE Industrial Electronics Society, IEEE, 2012, pp. 3221-3224.

[85] T. Geisel, V. Fairen, Statistical properties of chaos in chebyshev maps, Physics Letters A 105 (6) (1984) 263-266.

[86] Y. Mao, L. Cao, W. Liu, Design and fpga implementation of a pseudorandom bit sequence generator using spatiotemporal chaos, in: Communications, Circuits and Systems Proceedings, 2006 International Conference on, Vol. 3, IEEE, 2006, pp. 2114-2118.

[87] J. Černák, Digital generators of chaos, Physics letters A 214 (3) (1996) $151-160$.

[88] C.-Y. Li, J.-S. Chen, T.-Y. Chang, A chaos-based pseudo random number generator using timing-based reseeding method, in: Circuits and Systems, 2006. ISCAS 2006. Proceedings. 2006 IEEE International Symposium on, IEEE, 2006, pp. 4-pp.

[89] R. B, Simultaneous carry adder, uS Patent 2,966,305 (Dec. 27 1960). URL http: //www . google.com/patents/US2966305

[90] C.-Y. Li, Y.-H. Chen, T.-Y. Chang, L.-Y. Deng, K. To, Period extension and randomness enhancement using high-throughput reseeding-mixing prng, Very Large Scale Integration (VLSI) Systems, IEEE Transactions on 20 (2) (2012) 385-389.

[91] L.-Y. Deng, Efficient and portable multiple recursive generators of large order, ACM Transactions on Modeling and Computer Simulation (TOMACS) 15 (1) (2005) 1-13.

[92] T. Oshiba, Closure property of family of context-free languages under cyclic shift operation, ELECTRONICS \& COMMUNICATIONS IN JAPAN 55 (4) (1972) 119-122. 
[93] J. J. Shedletsky, Comment on the sequential and indeterminate behavior of an end-around-carry adder, IEEE Transactions on Computers 26 (3) (1977) 271-272.

[94] N. Hariprasad, et al., Fpga implementation of a cryptography technology using pseudo random number generator, in: International Journal of Engineering Research and Technology, Vol. 2, ESRSA Publications, 2013.

[95] O. Rssler, An equation for continuous chaos, Physics Letters A 57 (5) (1976) $397-398$. URL http://www.sciencedirect.com/science/article/pii/ 0375960176901018

[96] A. S. Elwakil, M. P. Kennedy, Construction of classes of circuitindependent chaotic oscillators using passive-only nonlinear devices, Circuits and Systems I: Fundamental Theory and Applications, IEEE Transactions on 48 (3) (2001) 289-307.

[97] A. Elwakil, M. Kennedy, Chaotic oscillator configuration using a frequency dependent negative resistor, International journal of circuit theory and applications 28 (1) (2000) 69-76.

[98] M. A. Zidan, A. G. Radwan, K. N. Salama, The effect of numerical techniques on differential equation based chaotic generators, in: Microelectronics (ICM), 2011 International Conference on, IEEE, 2011, pp. 1-4.

[99] G. Chen, J. Lü, Dynamics of the lorenz system family: analysis, control and synchronization, SciencePress, Beijing.

[100] P. Tsai, C. Merkle, T. Huang, Euler equation analysis of the propellerwake interaction, in: Symposium on Naval Hydrodynamics, 17th, 1900.

1160 [101] P. C. Hammer, The midpoint method of numerical integration, Mathematics Magazine 31 (4) (1958) 193-195. 
[102] J. C. Butcher, Numerical methods for ordinary differential equations in the 20th century, Journal of Computational and Applied Mathematics 125 (1) (2000) 1-29.

[108] J. M. Bahi, X. Fang, C. Guyeux, L. Larger, Fpga design for pseudorandom number generator based on chaotic iteration used in information hiding application, Appl. Math 7 (6) (2013) 2175-2188.

[109] Q. Wang, S. Yu, C. Guyeux, J. Bahi, X. Fang, Study on a new chaotic 1185 bitwise dynamical system and its FPGA implementation, Chinese Physics B 24 (6) (2015) 060503.

URL http://dx.doi.org/10.1088/1674-1056/24/6/060503

[110] Q. Wang, S. Yu, C. Guyeux, J. Bahi, X. Fang, A chaotic bitwise dynamical system and its FPGA-based realization, in: IWCFTA1'4, 7-th Int. 
Workshop on Chaos-Fractals Theories and Applications, Qingdao, China, 2014 , pp. ${ }^{* * *+* * * *}$.

[111] M. Bakiri, J.-F. Couchot, C. Guyeux, Fpga implementation of f2-linear pseudorandom number generators based on zynq mpsoc: A chaotic iterations post processing case study, in: Proceedings of the 13th International Joint Conference on e-Business and Telecommunications - Volume 4: SECRYPT,, 2016, pp. 302-309.

[112] M. Bakiri, J. F. Couchot, C. Guyeux, Ciprng: A vlsi family of chaotic iterations post-processings for $\mathrm{f}-2$ linear pseudorandom number generation based on zynq mpsoc, IEEE Transactions on Circuits and Systems I: Regular Papers PP (99) (2017) 1-14.

[113] M. Bakiri, J.-F. Couchot, C. Guyeux, One random jump and one permutation: Sufficient conditions to chaotic, statistically faultless, and large throughput prng for fpga, in: Proceedings of the 14th International Joint Conference on e-Business and Telecommunications - Volume 6: SECRYPT, (ICETE 2017), INSTICC, SciTePress, 2017, pp. 295-302.

[114] J.-F. Couchot, P.-C. Heam, C. Guyeux, Q. Wang, J. M. Bahi, Pseudorandom number generators with balanced gray codes, in: Security and Cryptography (SECRYPT), 2014 11th International Conference on, IEEE, 2014, pp. 1-7.

[115] S. Contassot-Vivier, J.-F. Couchot, C. Guyeux, P.-C. Heam, Random walk in a n-cube without hamiltonian cycle to chaotic pseudorandom number generation: Theoretical and practical considerations, International Journal of Bifurcation and Chaos 27 (01) (2017) 1750014.

[116] B. B. Purkayastha, K. K. Sarma, Digital phase-locked loop, in: A Digital Phase Locked Loop based Signal and Symbol Recovery System for Wireless Channel, Springer, 2015, pp. 103-126. 
[117] V. Fischer, M. Drutarovskỳ, True random number generator embedded in reconfigurable hardware, in: Cryptographic Hardware and Embedded Systems-CHES 2002, Springer, 2003, pp. 415-430.

[125] A. Cherkaoui, V. Fischer, A. Aubert, L. Fesquet, Comparison of self-timed ring and inverter ring oscillators as entropy sources in fpgas, in: Design,
Automation \& Test in Europe Conference \& Exhibition (DATE), 2012, ring and inverter ring oscillators as entropy sources in fpgas, in: Design,
Automation \& Test in Europe Conference \& Exhibition (DATE), 2012, IEEE, 2012, pp. 1325-1330.

[118] M. Šimka, M. Drutarovskỳ, V. Fischer, Embedded true random number generator in actel fpgas, in: Workshop on Cryptographic Advances in Secure Hardware-CRASH, 2005, pp. 6-7.

[119] M. Simka, M. Drutarovskỳ, V. Fischer, et al., Testing of pll-based true random number generator in changingworking conditions, RADIOENGINEERING, 20 (2011) 94-101.

[120] M. Varchola, M. Drutarovsky, R. Fouquet, V. Fischer, Hardware platform for testing performance of trngs embedded in actel fusion fpga, in: Radioelektronika, 2008 18th International Conference, IEEE, 2008, pp. $1-4$.

[121] P. Kohlbrenner, K. Gaj, An embedded true random number generator for fpgas, in: Proceedings of the 2004 ACM/SIGDA 12th international symposium on Field programmable gate arrays, ACM, 2004, pp. 71-78.

[122] C. Klein, O. Cret, A. Suciu, Design and implementation of a high quality and high throughput trng in fpga, arXiv preprint arXiv:0906.4762.

[123] M. Dichtl, J. D. Golić, High-speed true random number generation with logic gates only, Springer, 2007.

[124] A. Cherkaoui, V. Fischer, A. Aubert, L. Fesquet, A self-timed ring based true random number generator, in: Asynchronous Circuits and Systems (ASYNC), 2013 IEEE 19th International Symposium on, IEEE, 2013, pp. 99-106. 
[126] A. Cherkaoui, V. Fischer, L. Fesquet, A. Aubert, A very high speed true random number generator with entropy assessment, in: Cryptographic Hardware and Embedded Systems-CHES 2013, Springer, 2013, pp. 179196.

[127] I. E. Sutherland, Micropipelines, Communications of the ACM 32 (6)

[128] I. Vasyltsov, E. Hambardzumyan, Y.-S. Kim, B. Karpinskyy, Fast digital trng based on metastable ring oscillator, in: Cryptographic Hardware and Embedded Systems-CHES 2008, Springer, 2008, pp. 164-180.

[129] M. Majzoobi, F. Koushanfar, S. Devadas, Fpga-based true random num(1989) 720-738. ber generation using circuit metastability with adaptive feedback control, in: Cryptographic Hardware and Embedded Systems-CHES 2011, Springer, 2011, pp. 17-32.

[130] E. Salman, A. Dasdan, F. Taraporevala, K. Kucukcakar, E. G. Friedman, Exploiting setup-hold-time interdependence in static timing analysis, Computer-Aided Design of Integrated Circuits and Systems, IEEE Transactions on 26 (6) (2007) 1114-1125.

[131] D. Lee, H. Seo, H. Kim, Metastability-based feedback method for enhancing fpga-based trng., International Journal of Multimedia \& Ubiquitous Engineering 9 (3).

[132] NIST, National institute of standards and technology (nist): Fips140 - 1: Security requirements for cryptographic modules @ONLINE (1994). URL csrc.nist .gov/publications/fips/fips140-1/fips1401.pdf

[133] NIST, National institute of standards and technology (nist): A statistical test suite for random and pseudorandom number generators for cryptographic applications @ONLINE (2010).

URL csrc.nist.gov/publications/nistpubs/800-22-rev1a/ SP800-22rev1a.pdf 
[134] W. Killmann, W. Schindler, A proposal for: Functionality classes and evaluation methodology for true (physical) random number generators, TSystems debis Systemhaus Information Security Services and Bundesamt für Sicherheit in der Informationstechnik (BSI), Tech. Rep.

[135] NIST, National institute of standards and technology (nist): Fips140 - 2: Security requirements for cryptographic modules @ONLINE (May 2001). URL csrc.nist.gov/publications/fips/fips140-2/fips1402.pdf

[136] A. Canteaut, Berlekamp-massey algorithm, in: Encyclopedia of Cryptography and Security, Springer, 2011, pp. 80-80.

[137] I. Zarei Moghadam, A. S. Rostami, M. R. Tanhatalab, Designing a random number generator with novel parallel lfsr substructure for key stream ciphers, in: Computer Design and Applications (ICCDA), 2010 International Conference on, Vol. 5, IEEE, 2010, pp. V5-598.

[138] Z. Zhu, H. Hu, A dynamic nonlinear transform arithmetic for improving the properties chaos-based prng, in: Intelligent Control and Automation (WCICA), 2010 8th World Congress on, 2010, pp. 7055-7060.

[139] V. Bonato, B. F. Mazzotti, M. M. Fernandes, E. Marques, A mersenne twister hardware implementation for the monte carlo localization algorithm, Journal of Signal Processing Systems 70 (1) (2013) 75-85. 